\title{
Article \\ Symmetries and Selection Rules of the Spectra of Photoelectrons and High-Order Harmonics Generated by Field-Driven Atoms and Molecules
}

\author{
Dino Habibović ${ }^{1}\left(\mathbb{D}\right.$, Wilhelm Becker ${ }^{2,3, * \mathbb{C}}$ and Dejan B. Milošević ${ }^{1,2,4}(\mathbb{D}$ \\ 1 Faculty of Science, University of Sarajevo, Zmaja od Bosne 35, 71000 Sarajevo, Bosnia and Herzegovina; \\ dino.habibovic@pmf.unsa.ba or dhfizika1@gmail.com (D.H.); milo@bih.net.ba (D.B.M.) \\ 2 Max-Born-Institut, Max-Born-Strasse 2a, 12489 Berlin, Germany \\ 3 Department of Theoretical Nuclear Physics, National Research Nuclear University MEPhI, \\ Kashirskoe Shosse 31, 115409 Moscow, Russia \\ 4 Academy of Sciences and Arts of Bosnia and Herzegovina, Bistrik 7, 71000 Sarajevo, Bosnia and Herzegovina \\ * Correspondence: wbecker@mbi-berlin.de
}

Citation: Habibović, D.; Becker, W.; Milošević, D.B. Symmetries and Selection Rules of the Spectra of Photoelectrons and High-Order Harmonics Generated by Field-Driven Atoms and Molecules. Symmetry 2021, 13, 1566. https:// doi.org/10.3390/sym13091566

Academic Editor: Ossama Kullie

Received: 21 July 2021

Accepted: 12 August 2021

Published: 25 August 2021

Publisher's Note: MDPI stays neutral with regard to jurisdictional claims in published maps and institutional affiliations.

Copyright: (c) 2021 by the authors. Licensee MDPI, Basel, Switzerland. This article is an open access article distributed under the terms and conditions of the Creative Commons Attribution (CC BY) license (https:/ / creativecommons.org/licenses/by/ $4.0 /)$.

\begin{abstract}
Using the strong-field approximation we systematically investigate the selection rules for high-order harmonic generation and the symmetry properties of the angle-resolved photoelectron spectra for various atomic and molecular targets exposed to one-component and two-component laser fields. These include bicircular fields and orthogonally polarized two-color fields. The selection rules are derived directly from the dynamical symmetries of the driving field. Alternatively, we demonstrate that they can be obtained using the conservation of the projection of the total angular momentum on the quantization axis. We discuss how the harmonic spectra of atomic targets depend on the type of the ground state or, for molecular targets, on the pertinent molecular orbital. In addition, we briefly discuss some properties of the high-order harmonic spectra generated by a few-cycle laser field. The symmetry properties of the angle-resolved photoelectron momentum distribution are also determined by the dynamical symmetry of the driving field. We consider the first two terms in a Born series expansion of the $T$ matrix, which describe the direct and the rescattered electrons. Dynamical symmetries involving time translation generate rotational symmetries obeyed by both terms. However, those that involve time reversal generate reflection symmetries that are only observed by the direct electrons. Finally, we explain how the symmetry properties, imposed by the dynamical symmetry of the driving field, are altered for molecular targets.
\end{abstract}

Keywords: rotational symmetry; reflection symmetry; dynamical symmetry; above-threshold ionization; high-order harmonic generation; strong-field physics; atoms; molecules

\section{Introduction}

Symmetry is one of the most prominent concepts in physics, chemistry, and biology [1] Symmetries allow one to simplify a problem and predict a solution without actually solving it. Conserved quantities, which are the consequence of the symmetries of a system, can be obtained using Noether's theorem [2]. Symmetries are particularly important in the field of laser-matter interactions. When an atomic or molecular system is exposed to a strong laser field various nonlinear processes can take place (see the review papers [3-14] and references therein). While their detailed calculation may be cumbersome and require approximations, some features are determined solely by the symmetries of the atomic or molecular system and the driving laser field.

Among these processes above-threshold ionization (ATI) and high-order harmonic generation (HHG) are especially interesting and practically important. ATI is distinguished by the fact that it can be observed as a single-atom effect. In the ATI process the atom or molecule absorbs more photons from the laser field than is necessary for ionization so that the energy spectrum of the released electron consists of many peaks, which are 
separated by the photon energy. In addition, the electron once liberated may return to and rescatter off the parent ion. The rescattered electrons can gain additional kinetic energy from the laser field in a process sometimes called high-order above-threshold ionization (HATI). Alternatively, in the HHG process the released electron upon returning to its parent ion recombines into the ground state emitting its entire energy in the form of one high-energy photon with an energy equal to the kinetic energy gained in the laser field plus the ionization potential.

Depending on whether we use an atomic or a molecular target and on the type of the driving field some high-order harmonics may be absent from the spectra. This is determined by selection rules, which are related to the dynamical symmetry of the driving field and the symmetry properties of the target $[15,16]$. We speak of a dynamical symmetry if the total Hamiltonian, which governs the target exposed to the laser field, is left invariant under a combined time translation and spatial transformation such as rotation or reflection $[17,18]$. In addition to the total Hamiltonian, the symmetry of the ground-state wave function or, for molecular targets, the symmetry properties of the molecular orbitals may affect the selection rules. The symmetries of the (H)ATI photoelectron momentum spectra are also related to the dynamical symmetry of the laser field and the symmetries of the atomic or molecular system. Under dynamical symmetries we also subsume a symmetry operation that includes time reversal rather than time translation. As we will see, this may lead to approximate symmetries of the $(\mathrm{H})$ ATI angle-resolved spectra.

Over the past three decades, the HHG selection rules have been examined by various authors. The first examples of nonstandard driving fields leading to special selection rules for HHG other than the customary odd harmonics were bicircular fields investigated in [16,19-21] and orthogonally polarized two-color fields [16]. The combination of a fundamental circularly polarized field and another crossed linearly polarized secondharmonic field was proposed in [22]. The corresponding selection rules were derived using the dynamical symmetry of the Floquet Hamiltonian [17]. The selection rules for harmonic generation obtained using a bichromatic field with very similar frequencies $(\omega$ and $\omega+\delta \omega)$ of the field components were analyzed in [23]. In [24] it was shown how the selection rules for HHG of atomic targets exposed to a bicircular field are related to the dynamical symmetry of the field and, alternatively, how they can be derived using conservation of the projection of the angular momentum on the quantization axis. A similar problem was investigated in [25] where a bichromatic elliptically polarized field was employed to generate high-order harmonics. Again, the selection rules for atoms with closed shells were derived from the conservation of the projection of the angular momentum on the quantization axis. The generalization of these results to molecular targets and a bicircular field was presented in [26] for planar and [27] for nonplanar polyatomic molecules. Also, combining the symmetry properties of molecules and driving fields, the selection rules for HHG are obtained using time-dependent density functional theory in [28]. More recently, the selection rules for an orthogonally polarized two-color laser field were analyzed in [29]. The most general way to explore the relation between the symmetries and the selection rules is by using a group-theory approach. This was utilized in [30], and it is applicable to arbitrary molecules and laser-field configurations. Besides gaseous targets, harmonic generation can also be observed in a bulk crystal [31]. Here, the selection rules were derived taking into account only the interband currents, and it was shown that only odd harmonics can be expected in the spectrum. However, in [32] the intraband currents were considered as well, which breaks the selection rules so that both odd and even-order harmonics can be expected in the spectrum.

Above-threshold ionization of atomic and molecular targets exposed to various laser fields has also been analyzed in detail during the past decades (see [11,33-38] and references therein). In this case, the experimental features where symmetries become manifest include the shape and the spatial symmetries (reflection or rotation) of the angle-resolved spectrum of the liberated electron. In order to explain early experimental findings regarding the ATI plateau [39-44], S-matrix theory was applied for a contact potential $[45,46]$ and generalized 
to include the Coulomb potential [47]. This theory was applied to the ATI process in a bichromatic linearly polarized field in [48]. Rescattering by elliptically polarized driving fields was considered in $[49,50]$. From a symmetry point of view, the case where the atomic target is exposed to a two-component field is particularly interesting [51,52]. Examples are the bicircular and orthogonally polarized two-color (OTC) laser fields. The (H)ATI process in a bicircular field was analyzed theoretically in [53-55] and the calculated shape of the photoelectron spectrum was confirmed experimentally in [56]. The low-energy structures of the spectra observed in [56] were reproduced numerically, while their high-energy part was analyzed theoretically in [57] and experimentally in [58]. Moreover, the quantum-orbit theory for the HATI process in a bicircular field was developed in [59].

Besides atomic targets, molecules have also attracted a lot of attention [60,61]. They offer additional parameters, such as the Euler angles, which determine the position of the molecule with respect to the laser field, for control of the ionization. Rescattering and the characteristics of the photoelectron spectra are different from the case of atomic targets [62-69]. For example, in [70] it was shown that the molecular photoelectron distribution depends on the molecular alignment, the symmetry of the initial state and the carrier-envelope phase of the laser pulse. The photoelectron energy and momentum distributions for the ground state and the first-excited state of $\mathrm{H}_{2}^{+}$were analyzed in [71]. Moreover, ionization of the $\mathrm{H}_{2}^{+}$and $\mathrm{H}_{2}$ molecules driven by a circularly polarized attosecond XUV pulse was considered in [72], while the influence of the internuclear distance in the $\mathrm{H}_{2}^{+}$ion was examined in [73]. In [38] an extension of the strong-field approximation to the ionization of a diatomic molecule was formulated, and its generalization that includes rescattering, was given in [74-76]. Again, particularly interesting is the case where the molecular target is exposed to a two-component field. The symmetry properties of the photoelectron momentum spectra of homonuclear diatomic molecules exposed to a bicircular field were analyzed in [77], and linear triatomic molecules in the same field were considered in $[78,79]$. In addition, the interferences and plateau structures in the HATI spectra of homonuclear diatomic molecules in a bicircular field were analyzed in [80]. More recently, the symmetry properties of the (H)ATI spectra of homonuclear and heteronuclear diatomic molecules in OTC fields were scrutinized in [81,82].

To solve a quantum-mechanical problem two routes can be followed. The first route, based on the numerical solution of the time-dependent Schrödinger equation (TDSE) (see, for example [83-86] and references therein) becomes very demanding in computing power and time for all but the simplest problems. Hence, semianalytical theories can be beneficial. The numerical results presented in this paper are obtained using the approach based on the strong-field approximation (SFA), which relies on the fact that the applied laser field is so strong that the interaction of the released electron with its parent ion can be neglected, except for the eventual recombination or the rescattering process.

In this paper, we focus our attention on the relation between the laser-field dynamical symmetry and the symmetry of the target ground state on the one hand and the HHG selection rules and the symmetry properties of the (H)ATI spectra on the other. For illustration we use different atoms and molecules exposed to various monochromatic and bichromatic laser fields with various polarizations. The paper is partly a review of our own work with some new materials. It is organized as follows. In Section 2 we briefly discuss the SFA-based theory of the HHG and HATI processes, which we will employ. Also, we introduce the dynamical symmetries of various laser fields. Section 3 is devoted to symmetry considerations of the HHG selection rules, while in Section 4 we discuss the symmetries of the $(\mathrm{H})$ ATI photoelectron spectra imposed by the dynamical symmetry of the laser field and the symmetry of the ground state. Finally, in Section 5 we formulate our main conclusions. We use the atomic system of units.

\section{Theory}

In this section we present the framework for our analysis of the HHG selection rules and the symmetry properties of the photoelectron momentum distribution. 


\subsection{HHG Theory}

First, we briefly recall the theory of HHG that we will use. For a harmonic photon having frequency $\omega_{\mathbf{K}}=n \omega$ and unit complex polarization vector $\hat{\mathbf{e}}_{\mathbf{K}}$ the harmonic emission rate is given by [4]

$$
w_{n}\left(\omega_{\mathbf{K}}, \hat{\mathbf{e}}_{\mathbf{K}}\right)=\frac{\omega_{\mathbf{K}}^{3}}{2 \pi c^{3}}\left|\mathbf{T}_{n}\right|^{2},
$$

where $\mathbf{T}_{n}$ is the $T$-matrix element. It can be represented as the Fourier transform of the time-dependent dipole $\mathbf{d}(t)$

$$
\mathbf{d}(t)=\sum_{m} \mathbf{d}_{m}(t)
$$

in the form

$$
\mathbf{T}_{n}=\int_{0}^{T} \frac{d t}{T} e^{i n \omega t} \mathbf{d}(t) .
$$

For atomic targets, the sum over $m$ in Equation (2) is over the ground-state magnetic quantum number and the spin is neglected [24]. For atoms with an $s$ ground state (the example being $\mathrm{He}$ ) the sum over $m$ contains only the term $m=0$, while for atoms with a $p$ ground state (examples being the other noble gases) the sum over $m$ contains the three terms $m=0, \pm 1$. For molecular targets, the sum is over the relevant molecular orbitals [27]. Usually, only the highest occupied molecular orbital (HOMO) makes a significant contribution to the time-dependent dipole (2), but sometimes other molecular orbitals should also be taken into account [87].

In the strong-field approximation, the explicit form of the time-dependent dipole can be written as $[4,24,26,88]$

$$
\mathbf{d}_{m}(t)=C \int_{0}^{\infty} \frac{d \tau}{\tau^{3 / 2}} e^{-i S_{\mathrm{st}}\left(\mathbf{k}_{\mathrm{st}} ; t, t^{\prime}\right)} \mathcal{I}\left(\mathbf{k}_{\mathrm{st}}, t^{\prime}\right) \boldsymbol{\mathcal { R }}\left(\mathbf{k}_{\mathrm{st}}, t\right),
$$

where, for atomic targets, the ionization and recombination parts are

$$
\mathcal{I}\left(\mathbf{k}_{\mathrm{st}}, t^{\prime}\right)=\left\langle\mathbf{k}_{\mathrm{st}}+\mathbf{A}\left(t^{\prime}\right)\left|\mathbf{r} \cdot \mathbf{E}\left(t^{\prime}\right)\right| \psi_{\mathrm{i}}\right\rangle, \quad \mathcal{R}\left(\mathbf{k}_{\mathrm{st}}, t\right)=\left\langle\psi_{\mathrm{f}}|\mathbf{r}| \mathbf{k}_{\mathrm{st}}+\mathbf{A}(t)\right\rangle,
$$

respectively, while for molecular targets the corresponding expressions are

$$
\begin{aligned}
& \mathcal{I}\left(\mathbf{k}_{\mathrm{st}}, t^{\prime}\right)=\sum_{k=1}^{N} e^{-i \mathbf{k}_{\mathrm{st}} \cdot \mathbf{r}_{k}}\left\langle\mathbf{k}_{\mathrm{st}}+\mathbf{A}\left(t^{\prime}\right)\left|\mathbf{r} \cdot \mathbf{E}\left(t^{\prime}\right)\right| \psi_{k \mathrm{i}}\right\rangle, \\
& \mathcal{R}\left(\mathbf{k}_{\mathrm{st}}, t\right)=\sum_{j=1}^{N} e^{i\left[\mathbf{k}_{\mathrm{st}}+\mathbf{A}(t)\right] \cdot \mathbf{r}_{j}}\left\langle\psi_{j \mathrm{f}}|\mathbf{r}| \mathbf{k}_{\mathrm{st}}+\mathbf{A}(t)\right\rangle .
\end{aligned}
$$

Here $C=-i(2 \pi / i)^{3 / 2}, S_{\mathrm{st}}\left(\mathbf{k}_{\mathrm{st}} ; t, t^{\prime}\right)=\int_{t^{\prime}}^{t} d t^{\prime \prime}\left[\mathbf{k}_{\mathrm{st}}+\mathbf{A}\left(t^{\prime \prime}\right)\right]^{2} / 2+I_{p} \tau, I_{p}$ is the ionization potential, $\mathbf{A}(t)=-\int^{t} \mathbf{E}\left(t^{\prime \prime}\right) d t^{\prime \prime}$ the vector potential, $\mathbf{k}_{\mathrm{st}}=-\int_{t^{\prime}}^{t} \mathbf{A}\left(t^{\prime \prime}\right) d t^{\prime \prime} / \tau$ the stationary momentum between ionization and recombination, $t^{\prime}, t$ and $\tau=t-t^{\prime}$ are the ionization, recombination and travel time, respectively, and $\left|\psi_{j}\right\rangle(j=\mathrm{i}, \mathrm{f})$ are the initial and final atomic ground states. The molecular orbital is represented by a linear combination of atomic orbitals so that the sums over $k$ and $j$, which appear in Equation (6), are sums over the $N$ atomic centers. We use the linear combination of Slater-type orbitals obtained using the Hartree-Fock-Roothaan method to calculate the ground-state wave function of the atomic or diatomic molecular target, while for polyatomic molecules the atomic orbitals that construct the molecular orbital are represented by linear combinations of primitive Gaussians $[89,90]$. Finally, the coordinates $\mathbf{r}_{k}$ are those of the atomic centers as defined in $[26,91,92]$.

The so-called simple-man model can be used to get some information about the physics of the HHG process. In this model the motion of the electron in the laser field is treated classically using Newton's equation of motion $\ddot{\mathbf{r}}=-\mathbf{E}(t)$. The model is derived and applied to three-step processes in [39,93-95]. A two-dimensional generalization is given 
in $[96,97]$. To obtain the classical estimate of the cutoff position we demand that the kinetic energy $E_{\mathrm{k}}$ of the returning electron be extremal, i.e., $\partial E_{\mathrm{k}}\left(t, t^{\prime}\right) / \partial t^{\prime}=0$ and $\partial E_{\mathrm{k}}\left(t, t^{\prime}\right) / \partial t=0$. The solution of these equations gives the ionization and recombination times for which the electron's kinetic energy is extremal.

\subsection{HATI Theory}

Next, we briefly discuss the HATI theory. The differential ionization rate with absorption of $n$ photons for emission of a photoelectron with final momentum $\mathbf{p}$ can be expressed as [3]

$$
w_{\mathbf{p i}}(n)=2 \pi p\left|T_{\mathbf{p i}}(n)\right|^{2},
$$

where the $T$-matrix element consists of the contributions of the direct and the rescattered electrons. For atomic targets, these contributions are $[4,59]$

$$
\begin{gathered}
T_{\mathbf{p i}}^{(0)}(n)=\int_{0}^{T} \frac{d t^{\prime}}{T} e^{i\left[\mathcal{U}_{\mathbf{p}}\left(t^{\prime}\right)+n \omega t^{\prime}\right]}\left\langle\mathbf{p}+\mathbf{A}\left(t^{\prime}\right)\left|\mathbf{r} \cdot \mathbf{E}\left(t^{\prime}\right)\right| \psi_{\mathbf{i}}\right\rangle, \\
T_{\mathbf{p i}}^{(1)}(n)=C \int_{0}^{T} \frac{d t}{T} \int_{-\infty}^{t} \frac{d t^{\prime}}{\left(t-t^{\prime}\right)^{3 / 2}} e^{-i S_{\mathbf{p}, \mathrm{st}}\left(\mathbf{p}, \mathbf{k}_{\mathbf{s t}} ; t, t^{\prime}\right)}\left\langle\mathbf{p}|V(\mathbf{r})| \mathbf{k}_{\mathrm{st}}\right\rangle\left\langle\mathbf{k}_{\mathrm{st}}+\mathbf{A}\left(t^{\prime}\right)\left|\mathbf{r} \cdot \mathbf{E}\left(t^{\prime}\right)\right| \psi_{\mathrm{i}}\right\rangle,
\end{gathered}
$$

where $\mathcal{U}_{\mathbf{p}}(t)=\mathbf{p} \cdot \boldsymbol{\alpha}(t)+\int^{t} d t^{\prime \prime} \mathbf{A}^{2}\left(t^{\prime \prime}\right) / 2-U_{\mathrm{p}} t, \mathbf{A}(t)=d \boldsymbol{\alpha}(t) / d t, U_{\mathrm{p}}$ is the ponderomotive energy, $V(\mathbf{r})$ is the rescattering potential, $S_{\mathbf{p}, \mathrm{st}}\left(\mathbf{p}, \mathbf{k}_{\mathrm{st}} ; t, t^{\prime}\right)=S_{\mathrm{st}}\left(\mathbf{k}_{\mathrm{st}} ; t, t^{\prime}\right)-\mathcal{U}_{\mathbf{p}}(t)-n \omega t+$ $I_{p}\left(t-t^{\prime}\right)$ and $t$ is now the time of rescattering. The condition of energy conservation has the form $E_{\mathbf{p}}=\mathbf{p}^{2} / 2=n \omega-I_{p}-U_{\mathrm{p}}$.

Out of the four different versions of the molecular SFA considered in [38], the dressed modified molecular SFA in length gauge was confirmed to be the one most suitable $[98,99]$. It was extensively studied in [74-76], and the obtained results exhibited the best agreement with the experiments [100-104]. For molecular targets, the contributions of the direct and rescattered electrons are $[75,77]$

$$
\begin{aligned}
& T_{\mathbf{p i}}^{(0)}(n)=\int_{0}^{T} \frac{d t^{\prime}}{T} e^{i\left[\mathcal{U}_{\mathbf{p}}\left(t^{\prime}\right)+n \omega t^{\prime}\right]} \sum_{k=1}^{N} f\left(\mathbf{r}_{k}, t^{\prime}\right) e^{-i \mathbf{p} \cdot \mathbf{r}_{k}}\left\langle\mathbf{p}+\mathbf{A}\left(t^{\prime}\right)\left|\mathbf{r} \cdot \mathbf{E}\left(t^{\prime}\right)\right| \psi_{k \mathbf{i}}\right\rangle, \\
& T_{\mathbf{p i}}^{(1)}(n)= C \int_{0}^{T} \frac{d t}{T} \int_{-\infty}^{t} \frac{d t^{\prime}}{\left(t-t^{\prime}\right)^{3 / 2}} e^{-i S_{\mathbf{p}, \mathrm{st}}\left(\mathbf{p}, \mathbf{k}_{\mathrm{st}}: t, t^{\prime}\right)} \sum_{j=1}^{N} e^{i \mathbf{K}_{0} \cdot \mathbf{r}_{j}} V_{e \mathbf{K}_{0}}^{j} \\
& \times \sum_{l=1}^{N} f\left(\mathbf{r}_{l}, t^{\prime}\right) e^{-i \mathbf{k}_{\mathrm{st}} \cdot \mathbf{r}_{l}}\left\langle\mathbf{k}_{\mathrm{st}}+\mathbf{A}\left(t^{\prime}\right)\left|\mathbf{r} \cdot \mathbf{E}\left(t^{\prime}\right)\right| \psi_{l \mathrm{i}}\right\rangle
\end{aligned}
$$

respectively, where $\mathbf{K}_{0}=\mathbf{k}_{\mathrm{st}}-\mathbf{p}, V_{e \mathbf{K}_{0}}^{j}$ is the Fourier transform of the rescattering potential at the $j$ th center, and the function $f\left(\mathbf{r}_{l}, t\right)$ depends on the type of the molecular target [78]. For example, for linear symmetric molecules this function is equal to one.

\subsection{Laser Fields and Dynamical Symmetries}

We will use two coordinate systems, which are connected by the three Euler angles. The coordinate system in which the molecule is fixed is called the molecular coordinate system, while the laser field is defined in the so-called laser-field coordinate system. A general expression for the laser field $\mathbf{E}(t)=\left(E_{x}(t), E_{y}(t)\right)$ is

$$
\mathbf{E}(t)=\left(\mathcal{E}_{1} \sin \left(r \omega t+\varphi_{1}\right)-\kappa \varepsilon_{2} \mathcal{E}_{2} \cos \left(s \omega t+\varphi_{2}\right), \mathcal{E}_{2} \sin \left(s \omega t+\varphi_{2}\right)-\kappa \varepsilon_{1} \mathcal{E}_{1} \cos \left(r \omega t+\varphi_{1}\right)\right),
$$

where $\mathcal{E}_{j}=E_{j} / \sqrt{1+\varepsilon_{j}^{2}}$ and the dimensionless parameter $\kappa$ is used to distinguish different applied fields. The components incorporate two fields with intensities $I_{j}=E_{j}^{2}$, ellipticities $\varepsilon_{j}$ and phases $\varphi_{j}$ where $j=1,2$. The frequencies of these two fields are integer multiples 
of the fundamental frequency $\omega=2 \pi / T$ so that $r$ and $s$ are integers. We assume that $r$ and $s$ have no common divisors, i.e., $r+s$ even implies that both $r$ and $s$ are odd. For example, for $\kappa=1, r=1, \varphi_{1}=0, \varepsilon_{1}=\varepsilon$ and $\mathcal{E}_{2}=0$ the field (12) reduces to the elliptically polarized field

$$
\mathbf{E}(t)=\mathcal{E}_{1}(\sin \omega t,-\varepsilon \cos \omega t),
$$

for $\kappa=0, \varphi_{1}=0, \varphi_{2}=\varphi$ and $\varepsilon_{1}=\varepsilon_{2}=0$, it becomes the OTC field

$$
\mathbf{E}(t)=\left(E_{1} \sin (r \omega t), E_{2} \sin (s \omega t+\varphi)\right),
$$

and for $\kappa=1, \varphi_{1}=0, \varphi_{2}=\varphi+\pi / 2$ and $\varepsilon_{1}=\varepsilon_{2}=1$ we have the counter-rotating bicircular field

$$
\mathbf{E}(t)=\left(\mathcal{E}_{1} \sin (r \omega t)+\mathcal{E}_{2} \sin (s \omega t+\varphi), \mathcal{E}_{2} \cos (s \omega t+\varphi)-\mathcal{E}_{1} \cos (r \omega t)\right) .
$$

For the bicircular field, the relative phases $\varphi_{j}(j=1,2)$ are irrelevant because a change of one phase, with the other one fixed, leads to a rotation about the axis perpendicular to the laser-field polarization plane. In contrast, for the OTC field the value of the relative phase is crucial. It is common to fix $\varphi_{1}=0^{\circ}$ so that the other phase $\varphi=\varphi_{2}-\pi / 2$ is the relative phase between the field components. Examples of elliptically polarized (top panels), OTC (middle panels) and bicircular (bottom panels) fields (black solid lines), together with the corresponding vector potentials (red dashed lines), are shown in Figure 1. The arrows indicate the time evolution of the field and vector potential.

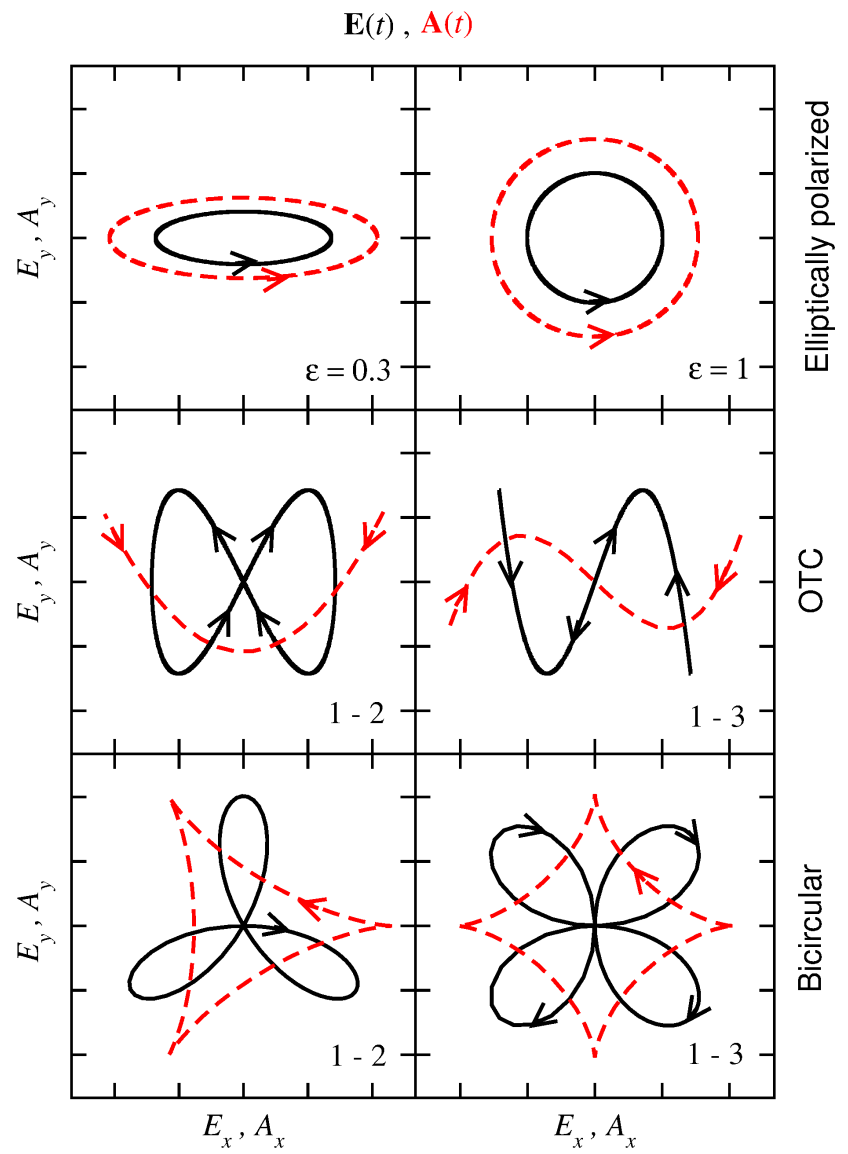

Figure 1. Examples of elliptically polarized (top panels), OTC (middle panels) and bicircular (bottom panels) fields (black solid lines), together with the corresponding vector potentials (red dashed lines). The ellipticity of the elliptically polarized field and the values of $r$ and $s$ for the OTC and bicircular fields are indicated in the panels. The relative phase between the OTC and the bicircular-field components is $\varphi=0^{\circ}$. The arrows indicate the time evolution of the field and the vector potential. 
We speak of a dynamical symmetry of the field, if a spatial transformation, such as a rotation by some angle or a reflection, can be compensated by a temporal transformation, such as a translation or a time reversal, so that the field including its time evolution remains unchanged. For example, for an elliptically polarized field, a rotation about the $z$ axis by the angle $\pi$ is compensated by a time translation by half a period:

$$
\mathbf{E}(t+T / 2)=-\mathbf{E}(t)=R_{z}(\pi) \mathbf{E}(t)
$$

regardless of the field ellipticity. Here $R_{z}(\pi)$ is the rotation matrix for rotation about the $z$ axis by the angle $\pi$. Similarly, for the OTC field we have [81]

$$
E_{x}(t+T / 2)=(-1)^{r} E_{x}(t), \quad E_{y}(t+T / 2)=(-1)^{s} E_{y}(t),
$$

which means that for even $r+s$ the dynamical symmetry is the same as for the elliptically polarized field, while for $r+s$ odd the time translation $t \rightarrow t+T / 2$ leads to the $x \rightarrow-x$ or $y \rightarrow-y$ reflection. This dynamical symmetry is unchanged if the field components are elliptically polarized. For the bicircular field, time translation by an integer multiple of the fraction $T /(r+s)$ of the field period corresponds to rotation by the angle $\alpha_{p}$ [24]:

$$
\mathbf{E}\left(t+\tau_{p}\right)=R_{z}\left(\alpha_{p}\right) \mathbf{E}(t)
$$

where $\tau_{p}=p T /(r+s), \alpha_{p}=-r \omega \tau_{p}$ and $p$ is an integer. The vector potentials obey the same symmetries (16)-(18) as the fields.

In addition to the dynamical symmetries that involve time translation and spatial rotation, the bottom panels of Figure 1 show that bicircular fields also observe reflection symmetries about several axes that are compensated by time reversal. For example, the bicircular field (15) with $r=1$ and $s=2$ satisfies the reflection symmetry:

$$
P_{x}(\pi / 3) \mathbf{E}(t)=-\mathbf{E}(T / 3-t)
$$

where $P_{x}(\beta)$ is the $2 \times 2$ matrix that effects a reflection in the $x-y$ plane about the axis that includes an angle $\beta$ with respect to the positive $x$ axis. This field possesses reflection symmetry with respect to two additional axes at the angles $\beta=\pi$ and $\beta=5 \pi / 3$ as well. However, for dynamical symmetries that imply time reversal, the vector potentials behave differently from the electric fields under a symmetry transformation: Equation (19) implies that the vector potentials transform as $P_{x}(\pi / 3) \mathbf{A}(t)=\mathbf{A}(T / 3-t)$. We will see below that symmetries that imply time reversal only give rise to approximate symmetries of the ionization amplitudes.

In the above examples we have assumed that the laser pulse is long, i.e., it consists of many optical cycles so that the amplitude can be considered as time-independent. However, few-cycle pulses, which consist of only a few optical cycles, have become widely available and now constitute indispensable tools. For such pulses, the amplitudes have to be considered as time-dependent. As an example, let us analyze the case of a linearly polarized few-cycle field with sine-square envelope, i.e., in Equation (12) we choose $r=1$, $\kappa=0, \varepsilon_{1}=0, E_{1}=E_{0} \sin ^{2}\left(\pi t / \tau_{p}\right), E_{2}=0, \varphi_{1}=\phi+\pi / 2$ where $\tau_{p}=n_{p} T$ is the length of the pulse expressed as a product of the number of optical cycles $n_{p}$ and the period $T=2 \pi / \omega$ and $\phi$ is the carrier-envelope phase. Introducing the notation $\omega_{p}=\omega / n_{p}$, $a=\left(n_{p}-1\right) / n_{p}$ and $b=\left(n_{p}+1\right) / n_{p}$, this field can be written as the trichromatic field

$$
E_{x}(t)=\frac{E_{0}}{4}[2 \cos (\omega t+\phi)-\cos (a \omega t+\phi)-\cos (b \omega t+\phi)] .
$$

As an example, in Figure 2 we present the few-cycle field (20) for $n_{p}=4$ as a function of the carrier-envelope phase and time. A comprehensive study of strong-field processes in a few-cycle field is given in [105], where the dependence of the HHG and HATI spectra on the carrier-envelope phase is particularly emphasized. In this context, we 
should mention a recently discovered high-order phase-dependent asymmetry in the HATI plateau [106], which is explained using an analogy with time-domain holography with electron wave packets.

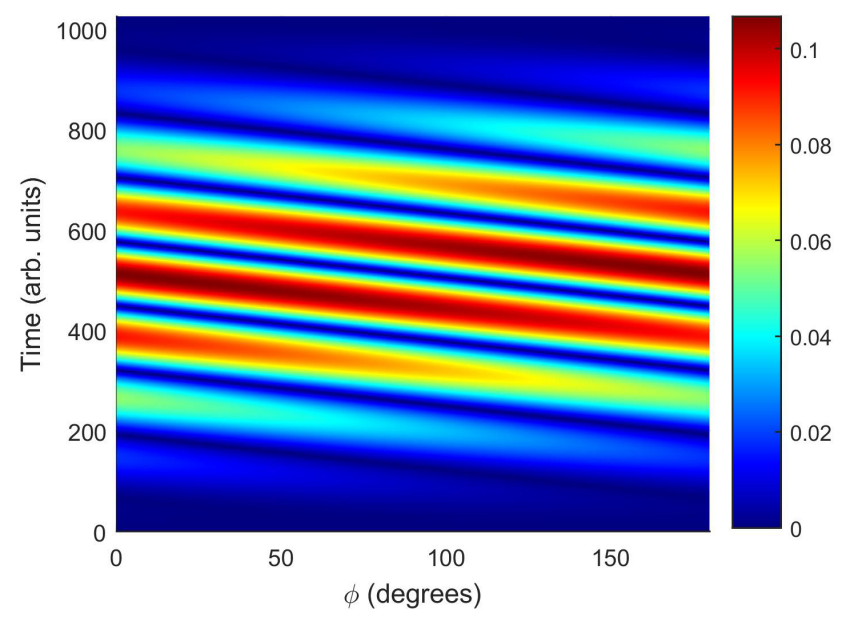

Figure 2. Intensity of a linearly polarized few-cycle pulse with $n_{p}=4$ and sine-square envelope as a function of the carrier-envelope phase and time.

\section{Selection Rules for HHG}

In this section we present several ways to obtain the HHG selection rules for fields that obey a dynamical symmetry. First, we derive the selection rules for atomic targets exposed to various laser fields and then we generalize them to the case of molecules. For illustrations, we use the atoms $\mathrm{He}$ and $\mathrm{Ar}$ as well as the molecules $\mathrm{O}_{3}$ and $\mathrm{CO}_{2}$. The helium atom has an $s$ ground state, while the ground state of the argon atom is $p$. On the other hand, the carbon dioxide molecule is an example of a linear molecule, while ozone is an example of a planar polyatomic molecule.

\subsection{Derivation of the Selection Rules}

We start with the simplest case of an elliptically polarized field, which satisfies the dynamical symmetry (16). The time-dependent dipole (2) can be written in the form $\mathbf{d}(t)=\left\langle\Psi_{\mathrm{f}}^{(-)}(t)|\mathbf{r}| \Psi_{\mathrm{i}}^{(+)}(t)\right\rangle$ where the states $\left|\Psi_{\mathrm{f}}^{(-)}(t)\right\rangle$ and $\left|\Psi_{\mathrm{i}}^{(+)}(t)\right\rangle$ satisfy the TDSE with the total Hamiltonian $H(t)$, which consists of the laser-free Hamiltonian $H_{0}$ and the interaction $\mathbf{r} \cdot \mathbf{E}$. This form is exact. Due to the dynamical symmetry of the elliptically polarized field the time-dependent dipole satisfies $\mathbf{d}(t+T / 2)=-\mathbf{d}(t)$ [20]. Since

$$
\mathbf{T}_{n}=\int_{0}^{T / 2} \frac{d t}{T} e^{i n \omega t}\left[\mathbf{d}(t)+\mathbf{d}(t+T / 2) e^{i n \omega T / 2}\right]
$$

only odd harmonics are emitted, i.e.,

$$
n=2 q \pm 1, \quad q=1,2, \ldots
$$

A similar procedure can be applied to obtain the selection rules for the bichromatic OTC field. The unitary rotation operator $D(\alpha)=e^{i \alpha J_{z}}$, where $J_{z}$ is the $z$ component of the total angular momentum, acts on the electron position operator according to $\mathbf{r}^{\prime}=R_{z}(\alpha) \mathbf{r}=$ $D(\alpha) \mathbf{r} D^{\dagger}(\alpha)$, while for the states $\left|\Psi_{j}^{( \pm)}(t)\right\rangle\left(j=\mathrm{i}\right.$, f) we have $\left|\Psi_{j}^{( \pm)}(t)\right\rangle=D(\alpha) \mid \Psi_{j}^{( \pm)}(t+$ $T / 2)\rangle$. Then, the time-dependent dipole satisfies $\mathbf{d}(t)=R_{z}(\alpha) \mathbf{d}(t+T / 2)$. For the OTC field with even $r+s$ the dynamical symmetry (17) shows that the time translation $t \rightarrow$ $t+T / 2$ corresponds to a rotation by the angle $\pi$ about the $z$ axis so that

$$
\mathbf{T}_{n}=\sum_{p=0,1} \int_{p T / 2}^{(p+1) T / 2} \frac{d t}{T} e^{i n \omega t} R_{z}(\pi) \mathbf{d}(t+p T / 2) .
$$


Using the substitution $t^{\prime}=t+p T / 2$ and $R_{z}(\pi) \mathbf{d}\left(t^{\prime}\right)=(-1)^{p} \mathbf{d}\left(t^{\prime}\right)(p=0,1)$ we get

$$
\mathbf{T}_{n}=\int_{0}^{T / 2} \frac{d t^{\prime}}{T} e^{i n \omega t^{\prime}} \sum_{p=0,1} e^{i n p \pi} R_{z}(\pi) \mathbf{d}\left(t^{\prime}\right)=\left[1-(-1)^{n}\right] \int_{0}^{T / 2} \frac{d t^{\prime}}{T} e^{i n \omega t^{\prime}} \mathbf{d}\left(t^{\prime}\right),
$$

which means that, for $r+s$ even only odd harmonics are present in the spectra and the selection rule (22) is valid again. For the OTC field with $r+s$ odd the dynamical symmetry is different, i.e., the time translation $t \rightarrow t+T / 2$ does not correspond to a rotation about the axis perpendicular to the polarization plane. Hence, both odd and even harmonics are emitted, i.e.,

$$
n=q \pm 1, \quad q=2,3, \ldots
$$

This derivation is unchanged if the field components are elliptically polarized. Such fields were analyzed in detail in terms of the high-order harmonic intensities and ellipticities as functions of the harmonic order in [107].

An analogous derivation of the selection rules for the case of a counter-rotating bicircular field was given in Appendix A of [24] and in [108]. A different but equivalent derivation was provided in [24]. There, the conservation of the projection of the total angular momentum on the quantization axis (in our case, the $z$ axis) is employed in order to obtain the selection rules. Let us now illustrate this procedure by using the counterrotating bicircular field as example. The conservation of the projection of the total angular momentum on the quantization axis can be written as

$$
\Delta J_{z, \mathrm{t}}+\Delta J_{z, \mathrm{l}}+\Delta J_{z, \mathrm{~h}}=0 .
$$

For the target atom we have $\Delta J_{z, \mathrm{t}}=m_{\mathrm{i}}-m_{\mathrm{f}}$ where $m_{\mathrm{i}}$ and $m_{\mathrm{f}}$ are the magnetic quantum numbers of the initial and final states. For the laser field, it is $\Delta J_{z, 1}=\Delta n_{r}-\Delta n_{s}$, where $\Delta n_{r}\left(\Delta n_{s}\right)$ is the difference between the number of absorbed and emitted photons of frequency $r \omega(s \omega)$. Because of energy conservation, the energy of the emitted harmonic is

$$
n \omega=\Delta n_{r} r \omega+\Delta n_{s} s \omega .
$$

Finally, a harmonic of ellipticity $\varepsilon_{n}= \pm 1$ is emitted so that $\Delta J_{z, \mathrm{~h}}=-\varepsilon_{n}$. Putting everything together, Equation (26) becomes

$$
m_{\mathrm{i}}-m_{\mathrm{f}}+\Delta n_{r}-\Delta n_{s}-\varepsilon_{n}=0,
$$

which together with Equation (27) leads to

$$
n \omega=q(r+s) \omega+\left(m_{\mathrm{f}}-m_{\mathrm{i}} \pm 1\right) r \omega,
$$

where we have introduced $q=\Delta n_{s}$. For atoms with an $s$ ground state $m_{\mathrm{f}}=m_{\mathrm{i}}=0$ so that the selection rule reads

$$
n \omega=q(r+s) \omega \pm r \omega .
$$

On the other hand, for atoms with a $p$ ground state the situation depends upon whether the shell is closed or not. In the former case, transitions that change the magnetic quantum number are forbidden by the Pauli exclusion principle, i.e., $m_{\mathrm{i}}=m_{\mathrm{f}}$ so that the selection rule is given by Equation (30), while in the latter case the general rule (29) has to be employed. A similar derivation of the corresponding selection rule is given in [107] for the OTC or, more generally, the bi-elliptical orthogonal two-color laser field.

The selection rules derived above were obtained using the $z$ axis as the quantization axis. They can be violated for a different choice of the quantization axis. For example, it is possible that the total Hamiltonian is invariant under a rotation by a certain angle, but this rotation is not a symmetry transformation of the ground state. A typical example is the atomic $p$ ground state, which is only invariant with respect to a rotation by an arbitrary angle about the $z$ axis. In contrast, the atomic $s$ ground state is invariant under 
rotations about any axis. This means that the derived selection rules are violated for atoms with a $p$ ground state if the quantization axis is not the $z$ axis (for more details see Appendix B in [24]). Of course, for atoms there is no preferred axis and we can always choose the $z$ axis as quantization axis so that the derived selection rules are valid for atoms. However, for molecules the internuclear axis is a preferred axis and the selection rules can be derived only for aligned molecules (see, for example, ref. [27]).

Let us now analyze what happens for molecular targets. In this case, for the derived selection rules to be satisfied, it is crucially important that the rotation that corresponds to a dynamical symmetry of the particular laser field be a symmetry transformation of the molecular ground state. To explain this in more detail we use the example of a symmetric linear molecule (e.g., $\mathrm{CO}_{2}$ ) placed in the field polarization plane. For the OTC field with $r+s$ even, the rotation that corresponds to the dynamical symmetry (17) (the rotation by the angle $\pi$ about the axis perpendicular to the polarization plane) is a symmetry transformation so that the selection rule (22) holds and only odd harmonics are emitted. In [28] it was shown that for molecules that are not aligned with the laser-field polarization plane the symmetry properties of the projection of the molecule on the polarization plane are relevant for the selection rules. For our example of the symmetric linear molecule this means that the selection rule (22) is valid even if the molecule is not aligned with the polarization plane due to the fact that its projection on the polarization plane is invariant with respect to the rotation imposed by the dynamical symmetry. Moreover, the selection rule (22) is also valid if the molecular projection on the polarization plane is invariant with respect to rotation by the angle $2 \pi /(2 k)$ (where $k$ is an integer) about the axis perpendicular to the polarization plane. A more comprehensive analysis in terms of the symmetries of the system molecule plus laser field is given in [28]. For the case where $r+s$ is odd both even and odd harmonics are present in the HHG spectra regardless of the molecular symmetry and orientation. A similar analysis can be done for other fields and more complex molecules.

\subsection{Illustration of the Selection Rules}

In this subsection we illustrate the obtained selection rules using the examples of $\mathrm{He}$ and $\mathrm{Ar}$ atoms and $\mathrm{O}_{3}$ and $\mathrm{CO}_{2}$ molecules. In Figure 3 we present the logarithm of the harmonic emission rate of the He atom as a function of the harmonic order. The left, middle and right panels correspond to elliptically polarized, OTC and bicircular fields, respectively. In the left panel, we display harmonic spectra for the linearly polarized (black dashed line, circles) as well as for the elliptically polarized field with the ellipticity $\varepsilon=0.1$ (red solid line, squares) and $\varepsilon=0.2$ (blue solid line, diamonds) and intensity $I=7 \times 10^{14} \mathrm{~W} / \mathrm{cm}^{2}$. Only odd harmonics are emitted as demanded by the selection rule (22). The harmonic emission rate quickly drops with increasing field ellipticity and the harmonic emission would be completely suppressed for a circularly polarized field [109]. In the middle panel of Figure 3 we exhibit spectra for the helium atom exposed to the OTC field with $(r, s)=(1,2)$ (black dashed line, circles) and $(r, s)=(1,3)$ (red solid line, squares). This case is governed by the selection rule (22) for $r+s$ even and the harmonics are elliptically polarized, while for odd $r+s$ the selection rule (25) applies and the harmonics are linearly polarized. Moreover, for odd $r+s$, the harmonic emission rates are significantly different for odd and even harmonics, while for $r+s$ even they vary smoothly with the harmonic order. Finally, in the right panel of Figure 3 we show spectra for a bicircular field with $(r, s)=(1,2)$ (black dashed line, circles) and $(r, s)=(1,3)$ (red solid line, squares), for the same component intensities as in the case of the OTC field. The He atom has an $s$ ground state so that the selection rule (30) has to be satisfied, which requires $n=3 q \pm 1(n=4 q \pm 1)$ for $r=1$ and $s=2(s=3)$. The emission rates of subsequent harmonics do not change very much as a function of the harmonic order, and the emitted harmonics are circularly polarized with alternating helicities. We chose component intensities below the saturation intensity so that consideration of saturation effects is not necessary. In the middle and right panels of Figure 3 two effects can be noticed. First, the cutoff energy is higher for 
the $(r, s)=(1,2)$ than for the $(r, s)=(1,3)$ case. The reason is that the cutoff energy is proportional to the ponderomotive energy which consists of the contributions of both laser-field components. These contributions are inversely proportional to the square of the frequency of the corresponding component, so that the contribution of the $2 \omega$ component is by the factor $9 / 4$ larger in energy than that of the $3 \omega$ component, which explains the obtained results. Second, the harmonic emission rate is much larger for the $(r, s)=(1,3)$ than for the $(r, s)=(1,2)$ case. This effect is more pronounced for the bicircular field. Explanation can be found in [110] (compare Figure 4 therein). The electric field vector of the $\omega-2 \omega$ field has three almost linear segments within one cycle, while the $\omega-3 \omega$ field has four such segments (see the lower panels of Figure 1). According to the quantum-orbit theory [20] the ionization (recombination) happens at the beginning (at the end) of these segments. Since more such segments are available for the $\omega-3 \omega$ than for the $\omega-2 \omega$ field, the corresponding harmonic emission rate is higher. In addition, the electron travel time $\tau$ of the dominant quantum orbit is shorter for the $\omega-3 \omega$ field. Since, according to semiclassical analysis from Refs. [20,110], the time-dependent dipole is proportional to $\tau^{-3 / 2}$ (physically, this is connected with wavepacket spreading), the emission rate is larger for the $\omega-3 \omega$ field. For the OTC field the results depend on the relative phase so that the above conclusion that the rates are much larger for the $\omega-3 \omega$ is not always fulfilled (compare Figure 1 in [111]).

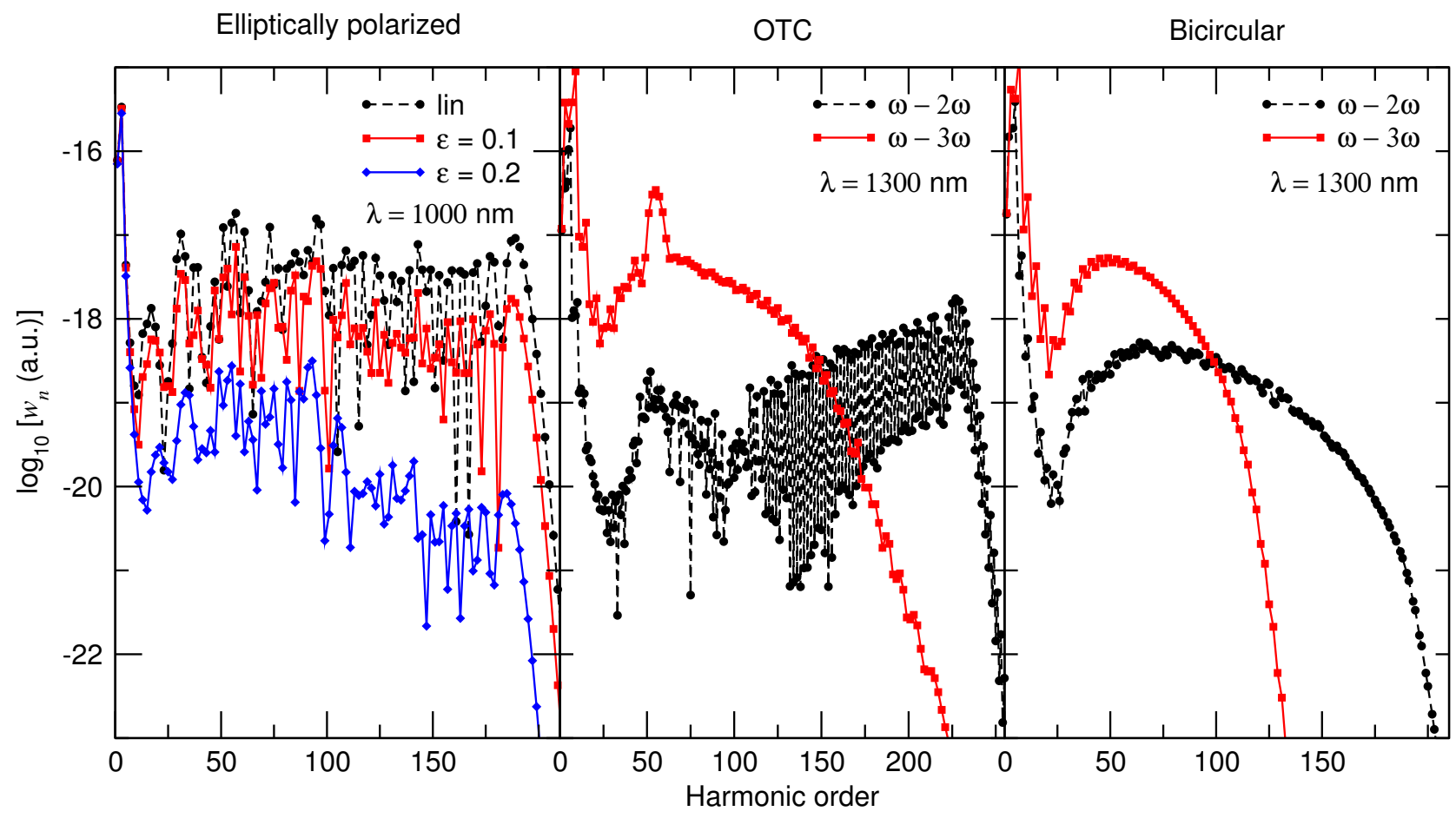

Figure 3. Logarithm of the harmonic emission rate of the He atom obtained using elliptically polarized (left panel, with denoted ellipticity $\varepsilon$ ), OTC (middle panel) and bicircular (right panel) fields. The intensity of the elliptically polarized field is $I=7 \times 10^{14} \mathrm{~W} / \mathrm{cm}^{2}$, while the component intensities of the $\omega-2 \omega$ and $\omega-3 \omega$ OTC and bicircular fields are equal, $I_{1}=I_{2}=I / 2$. The wavelength is indicated in each panel. The relative phase between the field components is $\varphi=0^{\circ}$.

Our second example is the Ar atom, which has a $p$ ground state. In Figure 4 we present the logarithm of the harmonic spectrum. Again, the left, middle and right panels correspond to elliptically polarized, OTC and bicircular fields, respectively. Now, the intensity of the elliptically polarized field is $I=2 \times 10^{14} \mathrm{~W} / \mathrm{cm}^{2}$ and for the OTC and bicircular field components we use $I_{1}=I_{2}=I / 2$. In order to obtain a longer plateau, we chose a longer wavelength than in the case of the He atom. The wavelength is indicated in each panel of Figure 4. The selection rules are the same as for helium. However, since Ar has a $p$ ground state, the quantization axis has to be the $z$ axis. The selection rules 
would be violated for a different quantization axis because the ground state would no longer be invariant with respect to a rotation about this axis. More about this can be found in $[24,112]$. Again, for the $\omega-2 \omega$ OTC field the harmonic emission rates of adjacent harmonics differ significantly. However, contrary to helium, for argon the emission rates of the odd harmonics generated by the $\omega-3 \omega$ OTC field do not change smoothly from one order to the next. This is due to the $p$ ground state of the Ar atom, which requires that the contributions of the magnetic quantum numbers $m=1$ and $m=-1$ be taken into account [113]. For the bicircular field as well, the main difference to He is that the emission rates of adjacent harmonics (with opposite helicities) are different. Depending on the harmonic order, the emission rate of the harmonics with helicity +1 can be higher or lower than those with helicity -1 . This behavior was explained in [24] in terms of the contributions with magnetic quantum numbers $m= \pm 1$, and it can be used for the generation of an elliptically polarized attosecond pulse [24,114,115].

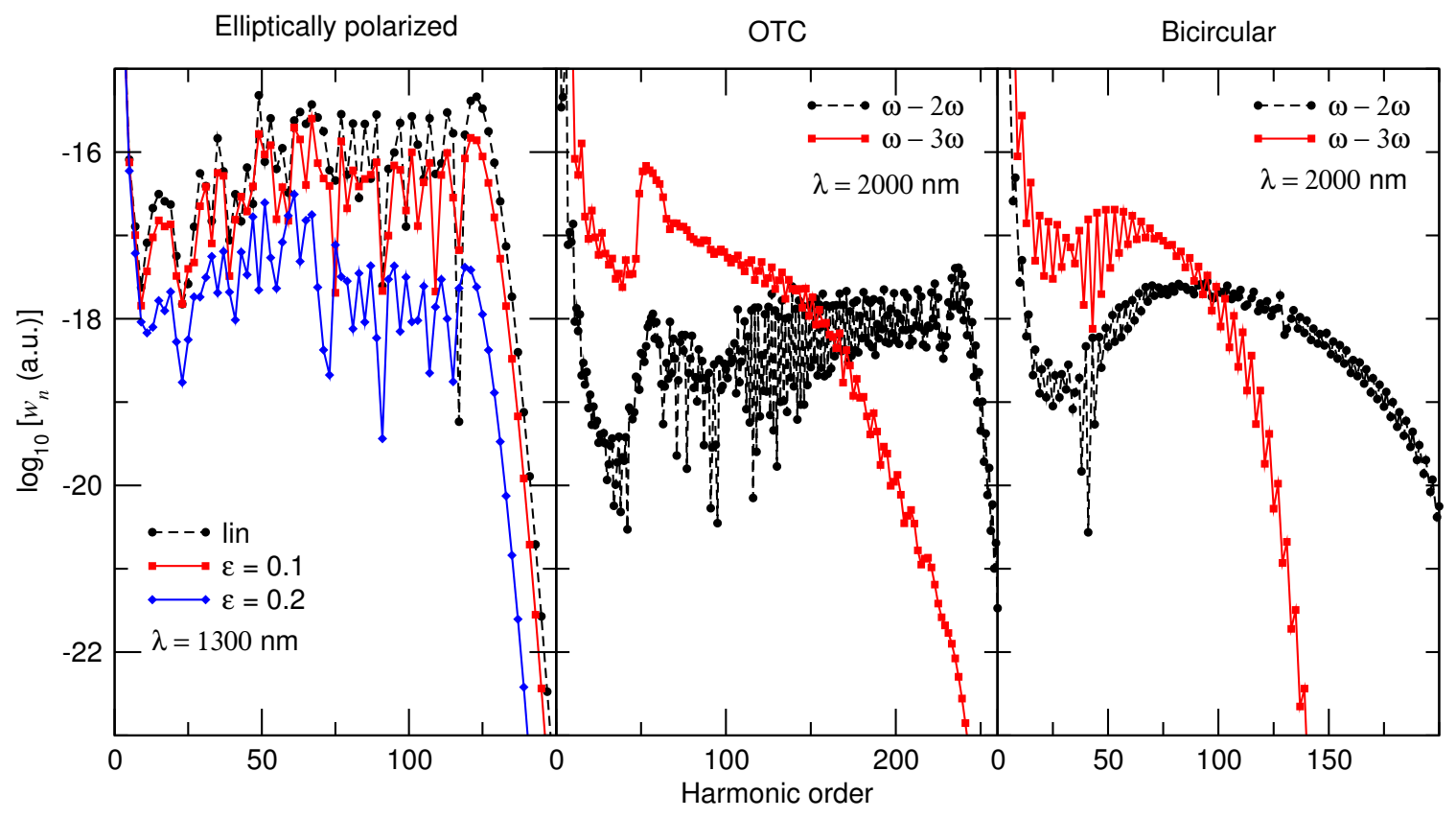

Figure 4. Logarithm of the harmonic emission rate of the Ar atom obtained using elliptically polarized (left panel, with denoted ellipticity $\varepsilon$ ), OTC (middle panel) and bicircular (right panel) fields. The intensity of the elliptically polarized field is $I=2 \times 10^{14} \mathrm{~W} / \mathrm{cm}^{2}$, while the intensities of the $\omega-2 \omega$ and $\omega-3 \omega$ OTC and the bicircular field components are equal $I_{1}=I_{2}=I / 2$. The wavelength is indicated in each panel. The relative phase between the field components is $\varphi=0^{\circ}$.

Having analyzed atomic targets let us now explore how the derived selection rules can be violated for molecular targets. As an example we use ozone, which is a planar molecule, exposed to the $\omega-3 \omega$ OTC field. We assume that the molecule is aligned with the polarization plane of the field. The spatial rotation about the axis perpendicular to the polarization plane by the angle $\pi$, which is implied by the dynamical symmetry (17) of the field, is not a symmetry transformation of the $\mathrm{O}_{3}$ molecule. Hence, a violation of the selection rule (22) is to be expected. To illustrate this, in Figure 5 we present the logarithm of the harmonic spectrum of the $\mathrm{O}_{3}$ molecule exposed to the $\omega-3 \omega$ OTC field (black dashed line, circles). Both odd and even harmonics are emitted so that the selection rule for atomic targets is now violated regardless of the orientation of the molecule in the polarization plane. The situation is similar and the selection rules are also violated, if the molecule is not aligned with the polarization plane. This could lead us to conclude that the selection rule (22) is also violated for a linear symmetric molecule that is not aligned with the polarization plane. However, this is not the case due to the fact that the symmetry properties of the projection of the molecule on the polarization plane are relevant instead 
of those of the molecule itself [28]. We discussed this problem already in the previous subsection. For an illustration, in Figure 5 we also present the harmonic spectrum of the $\mathrm{CO}_{2}$ molecule and the $\omega-3 \omega$ OTC field (red solid line, squares). The intensities of the field components are $I_{1}=I_{2}=6 \times 10^{13} \mathrm{~W} / \mathrm{cm}^{2}$, and the molecule is not aligned with the polarization plane (the values of the three Euler angles are $45^{\circ}$ ). Only odd harmonics are present in the spectrum, i.e., the selection rule (22) holds because the projection of the molecule on the polarization plane is invariant with respect to a rotation by the angle $\pi$ about the axis perpendicular to the polarization plane.

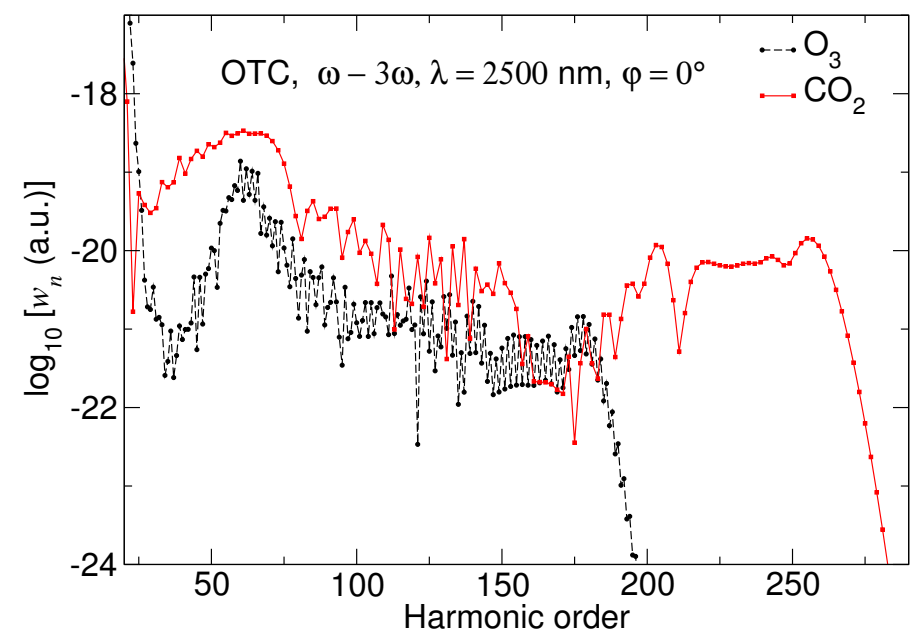

Figure 5. Logarithm of the harmonic emission rate of the $\mathrm{O}_{3}$ (black dashed line, circles) and $\mathrm{CO}_{2}$ (red solid line, squares) molecules for the $\omega-3 \omega$ OTC field. The field-component intensities are $I_{1}=I_{2}=4 \times 10^{13} \mathrm{~W} / \mathrm{cm}^{2}\left(I_{1}=I_{2}=6 \times 10^{13} \mathrm{~W} / \mathrm{cm}^{2}\right)$ for $\mathrm{O}_{3}\left(\mathrm{CO}_{2}\right)$ molecule. The other field parameters are indicated in the graph.

In [27] a similar analysis was performed for a nonplanar molecule exposed to a bicircular field using the example of the $\mathrm{NH}_{3}$ molecule, which is invariant with respect to a rotation about its principal axis by the angle $120^{\circ}$. Briefly, if the molecule is oriented such that its principal axis is perpendicular to the polarization plane and the dynamical symmetry of the field corresponds to a molecular symmetry, then the derived selection rule applies. The example is the $\omega-2 \omega$ bicircular field for which the transformation (18) corresponds to rotation by the angle $120^{\circ}$. This rotation is a symmetry transformation of the $\mathrm{NH}_{3}$ molecule. Finally, we mention that for nonplanar molecules harmonic emission with the propagation direction in the polarization plane can also be expected and the corresponding selection rule can be derived using the procedure shown above.

In the end, we briefly discuss the HHG spectrum generated by a few-cycle pulse, an example of which is given by Equation (20). The most important characteristic of such a spectrum is its pronounced dependence on the carrier-envelope phase. This effect was first demonstrated in [116] and experimentally observed for the first time in [117]. The local minima or maxima of the spectrum strongly depend on the value of the carrierenvelope phase. An example is given in Figure 2.6 of [105]. In addition, the position of the cutoff depends on the carrier-envelope phase and, according to the simple man's model introduced earlier, this position is lowest for $\phi=105^{\circ}$ [105].

\section{Symmetry Properties of the Photoelectron Momentum Distribution}

Having analyzed the connection between the dynamical symmetry and the groundstate symmetry on the one hand and the selection rules for harmonic emission on the other we will now investigate how these symmetries affect the photoelectron momentum distribution. The conclusions about the symmetry properties of the angle-resolved ATI and HATI spectra are illustrated using the $\mathrm{He}$ and $\mathrm{Ne}$ atoms as examples. 


\subsection{Symmetries of the Direct and Rescattered Photoelectron Momentum Spectra}

Again, for the OTC field, the cases where $r+s$ is even or odd have to be analyzed separately due to their different dynamical symmetries. We start our analysis with the $r \omega-s \omega$ OTC field and even $r+s$ so that the dynamical symmetry is given by Equation (17), i.e., the time translation $t \rightarrow t+T / 2$ corresponds to a rotation of the field by the angle $\pi$ about the axis perpendicular to the polarization plane. The matrix element

$$
F_{\mathbf{q}}\left(t^{\prime}\right)=\left\langle\mathbf{q}+\mathbf{A}\left(t^{\prime}\right)\left|\mathbf{r} \cdot \mathbf{E}\left(t^{\prime}\right)\right| \psi_{\mathrm{i}}\right\rangle=\int \frac{d \mathbf{r}}{(2 \pi)^{3 / 2}} e^{-i\left[\mathbf{q}+\mathbf{A}\left(t^{\prime}\right)\right] \cdot \mathbf{r}} \mathbf{r} \cdot \mathbf{E}\left(t^{\prime}\right) \psi_{\mathrm{i} l m}(\mathbf{r})
$$

appears in both the direct (Equation (8)) and the rescattered (Equation (9)) contribution to the $T$ matrix for atomic targets. For the direct electrons $\mathbf{q}=\mathbf{p}$, while for the rescattered electrons $\mathbf{q}=\mathbf{k}_{\mathrm{st}}$. In either case, $\left|\psi_{\mathrm{i}}\right\rangle$ is the atomic orbital of the initial state. For the momentum $\mathbf{q}$ rotated by $\pi$ about the $z$ axis, i.e., $\mathbf{q}^{\prime}=R_{z}(\pi) \mathbf{q}$, we can easily see that

$$
F_{\mathbf{q}^{\prime}}(t)=e^{-i m \pi} F_{\mathbf{q}}(t+T / 2),
$$

where the factor $\exp (-i m \pi)$ comes from the behavior of the initial-state wave function under the rotation $\mathbf{r}^{\prime}=R_{z}(\pi) \mathbf{r}$. The matrix elements of the direct and the rescattering parts of the $T$ matrix also contain the terms $\mathcal{U}_{\mathbf{p}}\left(t^{\prime}\right)+n \omega t^{\prime}$ and $S_{\mathbf{p}, \text { st }}\left(\mathbf{p}, \mathbf{k}_{\mathrm{st}} ; t, t^{\prime}\right)$, respectively. The transformation rules for these terms are $\mathcal{U}_{\mathbf{p}}\left(t^{\prime}\right)+n \omega t^{\prime}=\mathcal{U}_{\mathbf{p}^{\prime}}\left(t^{\prime}+T / 2\right)+n \omega t^{\prime}+n \omega T / 2$ and $S_{\mathbf{p}, \mathrm{st}}\left(\mathbf{p}, \mathbf{k}_{\mathrm{st}} ; t, t^{\prime}\right)=S_{\mathbf{p}^{\prime}, \mathrm{st}}\left(\mathbf{p}^{\prime}, \mathbf{k}_{\mathrm{st}}^{\prime} ; t+T / 2, t^{\prime}+T / 2\right)$. Recalling the form of the function $\mathcal{U}_{\mathbf{p}}(t)$ we get

$$
T_{\mathbf{p i}}^{(0)}(n)+T_{\mathbf{p i}}^{(1)}(n)=e^{i\left(n \omega-m-U_{p}\right) T / 2}\left[T_{\mathbf{p}^{\prime} \mathbf{i}}^{(0)}(n)+T_{\mathbf{p}^{\prime} \mathbf{i}}^{(1)}(n)\right] .
$$

Hence, for the OTC field with $r+s$ even, the $T$-matrix elements for two momenta that are connected by the rotation corresponding to the dynamical symmetry (17) only differ by a phase so that they yield identical spectra. An illustration is provided by the middle panel of Figure 6. Recall that $T_{\mathbf{p i}}^{(0)}(n)+T_{\mathbf{p i}}^{(1)}(n)$ are just the first two terms of the Born series. In the Appendix $\mathrm{A}$ it is shown that the rotational symmetry holds for all orders of the Born series.

For $r+s$ odd, the dynamical symmetry (17) implies a reflection with respect to the $x z$ or the $y z$ plane ( $y \rightarrow-y$ or $x \rightarrow-x$, depending on whether $r$ or $s$ is even), in place of the rotation for $r+s$ even. Indeed, an analysis similar to the one above, now predicts reflection symmetries. The left and right panels of Figure 6 present examples.

It is important to notice that Figure 6 displays the contributions of the direct and the rescattered electrons taken together. Both satisfy the same symmetries. For the OTC fields, the spectra exhibit the symmetries regardless of the values of the relative phase; only the quantitative values of the differential ionization rates depend on the relative phase. For example, in the left (right) panels of Figure 7 we present similar results as in the left (middle) panel of Figure 6 but for the OTC field with the relative phases $\varphi=30^{\circ}$ (upper row) and $\varphi=90^{\circ}$ (lower row).

The spectra shown in Figures 6 and 7 all consist of a central part with high yield, which is due to the direct electrons, and surrounding discs with much lower yields, which are due to rescattering. The disc-like distributions are well known from the case of linear polarization [118] and are also familiar for bicircular fields [58]. Close inspection of the $\omega-3 \omega$ spectra in the right panels of Figure 7 reveals some features reminiscent of smoke trails, which emanate from the central part in a counterclockwise direction. They are likely due to interference of the contributions of the direct and the rescattered electrons just like the spiders observed for linear polarization [119-121]. Very similar effects are also visible for bicircular fields [59,122]. 

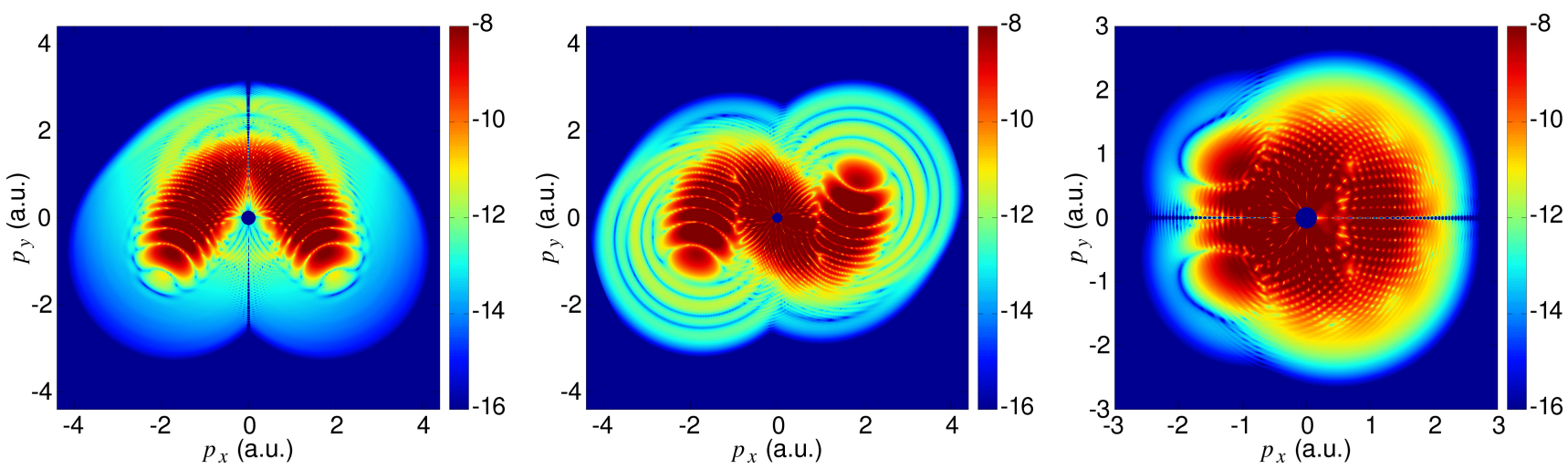

Figure 6. Logarithm of the differential ionization rate of the He atom exposed to the $\omega-2 \omega$ (left panel), $\omega-3 \omega$ (middle panel) and $2 \omega-3 \omega$ (right panel) OTC fields with the relative phase $\varphi=0^{\circ}$. The intensities of the field components and the fundamental wavelength are $I_{1}=I_{2}=3.5 \times 10^{14} \mathrm{~W} / \mathrm{cm}^{2}$ and $\lambda=800 \mathrm{~nm}$. The contributions of both direct and rescattered electrons are taken into account.
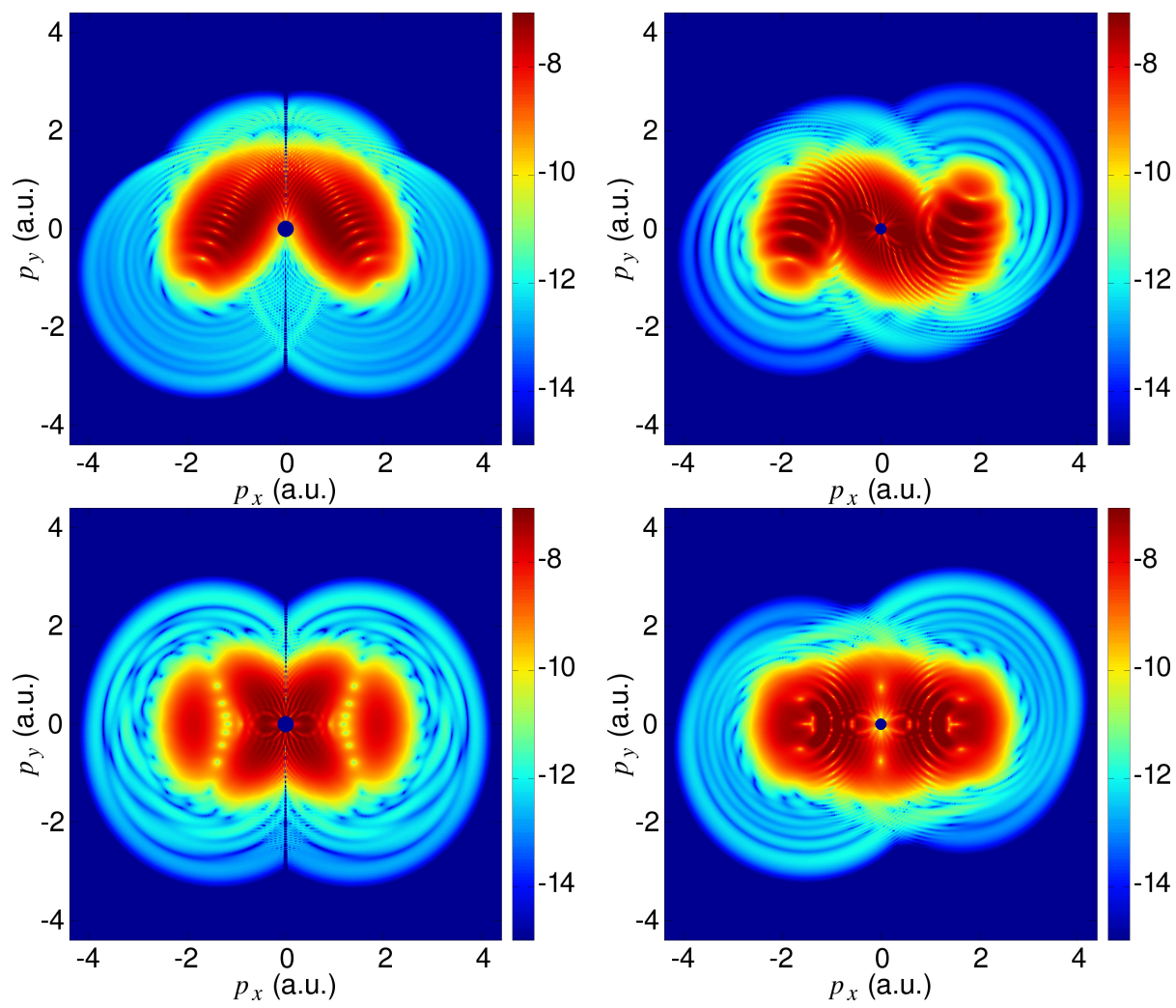

Figure 7. Logarithm of the differential ionization rate of the He atom exposed to the $\omega-2 \omega$ (left panels) and $\omega-3 \omega$ (right panels) OTC fields with the relative phases $\varphi=30^{\circ}$ (upper row) and $\varphi=90^{\circ}$ (lower row). The contributions of both direct and rescattered electrons are taken into account. The other laser-field parameters are the same as in Figure 6.

The angle-dependent spectrum for the $\omega-3 \omega$ field with $\varphi=90^{\circ}$ in the lower panels of Figure 7 displays an additional approximate symmetry, viz., reflection with respect to the $x z$ plane $(y \rightarrow-y)$, which is obeyed only by the direct electrons. This will be discussed in the next subsection.

Bicircular fields whose dynamical symmetry is given by Equation (18) provide particularly interesting angle-resolved spectra, which strongly depend on the frequencies of the field components. In [59] it was shown that the rotation by the angle $\alpha_{p}$ only leads to 
a phase factor, which is the same for the direct and the rescattering term of the $T$-matrix element, i.e.,

$$
T_{\mathbf{p i}}^{(0)}(n)+T_{\mathbf{p i}}^{(1)}(n)=e^{i \gamma}\left[T_{\mathbf{p}^{\prime} \mathbf{i}}^{(0)}(n)+T_{\mathbf{p}^{\prime} \mathbf{i}}^{(1)}(n)\right],
$$

where $\gamma=-m r \omega \tau_{p}-n \omega \tau_{p}$. This implies that the photoelectron momentum distribution is invariant with respect to a rotation by the angle $\alpha_{p}$ around the axis perpendicular to the polarization plane. For example, for $r=1$ and $s=2$ the spectrum is invariant with respect to the rotation by the angle $\alpha_{p}=-2 p \pi / 3$. This is exhibited in Figure 5 of [59], along with other examples for different combinations of $r$ and $s$.

Finally, in order to inspect the influence of the type of the ground state on the symmetries of the HATI spectra, in Figure 8 we present the same results as in Figure 6 but for the Ar atom which has a $p$ ground state. The intensities of the field components and the fundamental wavelength are $I_{1}=I_{2}=2 \times 10^{14} \mathrm{~W} / \mathrm{cm}^{2}$ and $\lambda=800 \mathrm{~nm}$. Clearly, the symmetry properties are the same as for the He atom. Only the differential ionization rate for Ar is higher because of the lower value of the ionization potential.
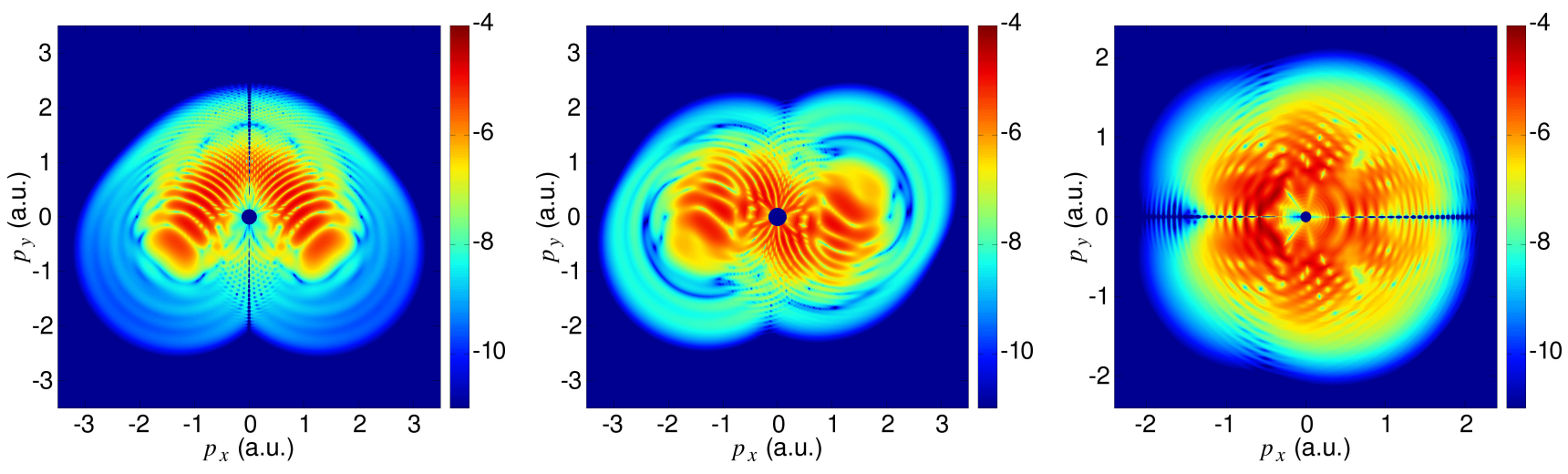

Figure 8. The same as in Figure 6 but for the Ar atom. The intensities of the field components and the fundamental wavelength are $I_{1}=I_{2}=2 \times 10^{14} \mathrm{~W} / \mathrm{cm}^{2}$ and $\lambda=800 \mathrm{~nm}$.

Concluding, the ATI and HATI photoelectron momentum spectra obey symmetry properties that are directly imposed by the dynamical symmetry of the applied field. This holds regardless of the type of the atomic ground state.

\subsection{Symmetries of the Direct Photoelectron Momentum Spectra}

So far, we have analyzed the symmetries of the angle-resolved ionization spectra of the first two terms of the Born series of the $T$ matrix. However, the spectrum of the lower right panel of Figure $7\left(\omega-3 \omega\right.$ OTC with a relative phase of $\left.90^{\circ}\right)$ suggests that the spectrum of the direct electrons, which dominates the low-energy part of the spectrum, may exhibit additional symmetries. We will now look into this more closely.

As a first example, we consider the $r \omega-s \omega$ bicircular field for which a graph of the vector potential (disregarding its time evolution) obeys reflection symmetry about the axes at the angles $\beta_{p}=-\alpha_{p} / 2$ with respect to the positive $x$ axis, $\mathrm{cf}$. the bottom panels of Figure 1. More precisely, denoting the transformed vectors by a double prime, we have

$$
\mathbf{A}^{\prime \prime}(t) \equiv P_{x}\left(\beta_{1}\right) \mathbf{A}(t)=\mathbf{A}\left(\tau_{1}-t\right),
$$

where $P_{x}\left(\beta_{1}\right)$ is the $2 \times 2$ reflection matrix that generates a reflection in the $x-y$ plane about the axis that includes the angle $\beta_{1}$ with the positive $x$ axis. Its off-diagonal elements are $\sin \left(2 \beta_{1}\right)$ and the diagonal elements are $\left[P_{x}\left(\beta_{1}\right)\right]_{j j}=(-1)^{j+1} \cos \left(2 \beta_{1}\right)$. Due to the $(r+s)$-fold rotational symmetry it is enough to consider the reflection symmetry for the angle $\beta_{1}=\pi r /(r+s)$. In (35) the reflection is compensated by a time translation by $\tau_{1}=T /(r+s)$ plus time reversal $t \rightarrow-t$. Since $\mathbf{E}(t)=-d \mathbf{A}(t) / d t$ the electric-field vector changes its sign under this reflection, i.e., $\mathbf{E}^{\prime \prime}(t)=-\mathbf{E}\left(\tau_{1}-t\right)$ (compare the special case 
given by Equation (19)). It was shown numerically in [59] that the direct ionization rate is invariant with respect to this transformation, i.e.

$$
w_{\mathbf{p}^{\prime \prime i}}^{(0)}=w_{\mathbf{p i}}^{(0)}, \quad \mathbf{p}^{\prime \prime}=P_{x}\left(\beta_{1}\right) \mathbf{p}
$$

for the direct electrons.

In order to prove Equation (36) we express the reflection matrix $P_{x}\left(\beta_{1}\right)$ through the matrices $R_{z}\left(\beta_{1}\right)$ and $P_{x}(0)$ as $P_{x}\left(\beta_{1}\right)=R_{z}\left(\beta_{1}\right) P_{x}(0) R_{z}\left(-\beta_{1}\right)$. The corresponding operator in Hilbert space is $e^{i \beta_{1} J_{z}} \hat{P}_{y} e^{-i \beta_{1} J_{z}}$ where the operator $\hat{P}_{y}$ corresponds to the reflection $y \rightarrow$ $-y$, which is performed by the matrix $P_{x}(0)$. Since the Hamiltonian $H_{0}+V$ is invariant with respect to the reflection that corresponds to $P_{x}\left(\beta_{1}\right)$, for the ground-state wave function we obtain $\psi_{\mathrm{i} l m}(\mathbf{r})=\psi_{\mathrm{i} l m}^{\prime \prime}\left(\mathbf{r}^{\prime \prime}\right)$ and $\psi_{\mathrm{i} l m}^{\prime \prime}(\mathbf{r})=e^{i \beta_{1} J_{z}} \hat{P}_{y} e^{-i \beta_{1} J_{z}} \psi_{\mathrm{i} l m}(\mathbf{r})=e^{i \beta_{1}\left(J_{z}-m\right)} \hat{P}_{y} \psi_{\mathrm{i} l m}(r, \theta, \phi)$. Since $y \rightarrow-y$ corresponds to $\phi \rightarrow-\phi$ and since for the spherical harmonics we have $Y_{l m}(\theta,-\phi)=Y_{l m}^{*}(\theta, \phi)=(-1)^{m} Y_{l,-m}(\theta, \phi)$, we get $\psi_{\mathrm{i} l m}^{\prime \prime}(\mathbf{r})=e^{-2 i m \beta_{1}} \psi_{\mathrm{i} l m}^{*}(\mathbf{r})$. On the other hand, the spatial inversion property of the spherical harmonics $Y_{l m}(\pi-\theta, \pi+\phi)=$ $(-1)^{l} Y_{l m}(\theta, \phi)$ leads to

$$
\psi_{\mathrm{i} l m}(\mathbf{r})=\psi_{\mathrm{i} l m}^{\prime \prime}\left(\mathbf{r}^{\prime \prime}\right)=e^{-2 i m \beta_{1}}(-1)^{l}\left[\psi_{\mathrm{i} l m}\left(-\mathbf{r}^{\prime \prime}\right)\right]^{*} .
$$

Using (37) we obtain $F_{\mathbf{p}}\left(t^{\prime}\right)=(-1)^{l} e^{-2 i m \beta_{1}}\left[F_{\mathbf{p}^{\prime \prime}}\left(\tau_{1}-t^{\prime}\right)\right]^{*}$ and $\mathcal{U}_{\mathbf{p}}\left(t^{\prime}\right)+n \omega t^{\prime}=-\mathcal{U}_{\mathbf{p}^{\prime \prime}}$ $\left(\tau_{1}-t^{\prime}\right)-n \omega\left(\tau_{1}-t^{\prime}\right)+\left(n \omega-U_{p}\right) \tau_{1}$ so that

$$
T_{\mathbf{p i}}^{(0)}(n)=(-1)^{l} e^{i\left(n \omega-U_{p}\right) \tau_{1}-2 i m \beta_{1}}\left[T_{\mathbf{p}^{\prime \prime} \mathbf{i}}^{(0)}(n)\right]^{*},
$$

which implies that Equation (36) is valid, i.e., the direct photoelectron momentum spectra are invariant under the reflection $P_{x}\left(\beta_{1}\right)$. Examples of this symmetry are shown in Figure 2 of [59] for various combinations of $r$ and $s$.

Our second example concerns the OTC field with $r+s$ even, for which a similar procedure can be carried out. While the transformation of the wave function is unchanged, the main difference occurs because the OTC field does not, in general, satisfy a relation similar to Equation (35). As a result, the direct-electron ATI spectra do not, in general, possess any additional symmetries. Let us now illustrate these considerations with a few numerical examples. In Figure 9 we present the logarithm of the differential ionization rate of the He atom exposed to the $\omega-2 \omega$ (left panel), $\omega-3 \omega$ (middle panel) and $2 \omega-3 \omega$ (right panel) OTC fields with the relative phase $\varphi=0^{\circ}$. Only the contribution of the direct electrons is taken into account.
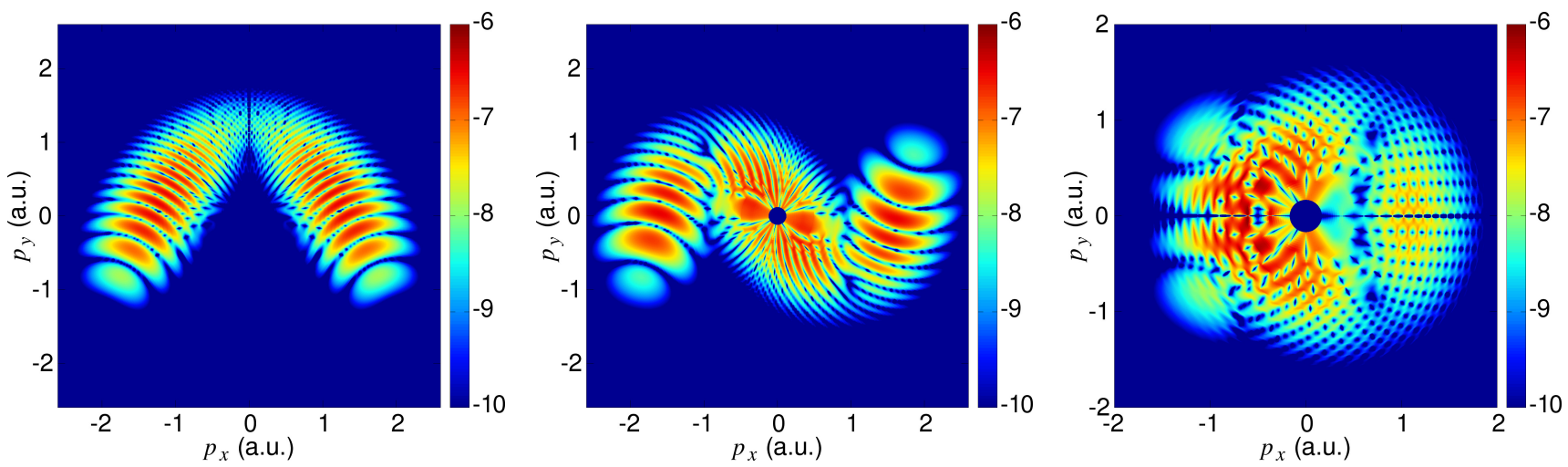

Figure 9. Logarithm of the differential ionization rate of the He atom exposed to the $\omega-2 \omega$ (left panel), $\omega$-3 $\omega$ (middle panel) and $2 \omega-3 \omega$ (right panel) OTC fields with the relative phase $\varphi=0^{\circ}$. The spectra only display the direct electrons; they are the analogs of those in Figure 6 where both direct and rescattered eletrons are taken into account. The intensity of the field components and the fundamental wavelength are $I_{1}=I_{2}=3.5 \times 10^{14} \mathrm{~W} / \mathrm{cm}^{2}$ and $\lambda=800 \mathrm{~nm}$. 
In contrast to the previous case of the bicircular field, the direct photoelectron spectra obtained using the OTC field with an arbitrary relative phase possess only the rotational symmetry imposed by the dynamical symmetry involving time translation of the corresponding field. In the particular case of Figure 9, the spectrum calculated using the $\omega-2 \omega$ $(2 \omega-3 \omega)$ OTC field exhibits reflection symmetry with respect to the $y z(x z)$ plane, i.e., $x \rightarrow-x(y \rightarrow-y)$, while for the $\omega-3 \omega$ OTC field it is invariant under the rotation about the $z$ axis by the angle $\pi$. The spectra for $\omega-2 \omega$ and $\omega-3 \omega$ OTC fields shown in the left and middle panels of Figure 9 can be compared with the corresponding vector potentials plotted in Figure 1. As should be, the envelopes of $-\mathbf{A}(t)$ essentially reproduce the former.

Finally, we will focus on the fact, already noticed above in the lower panels of Figure 7, that for some values of the relative phase an additional symmetry can be present in the direct spectra, which are generated by $T_{\mathbf{p i}}^{(0)}(n)$. This symmetry is violated by the rescattered spectra generated by $T_{\mathrm{pi}}^{(1)}(n)$. As an example, in Figure 10 we present spectra for the same parameters as in the lower row of Figure 7, but this time only taking into account the direct electrons. For the OTC field with $\varphi=90^{\circ}$, these spectra possess reflection symmetry with respect to the $x z$ plane $(y \rightarrow-y)$. For $r+s$ odd, this reflection symmetry is not shared by the rescattered electrons so that the entire spectrum exhibits only reflection symmetry with respect to the $y z$ plane. Also, for $r+s$ even, the rescattered electrons exhibit only the rotational symmetry by the angle $\pi$ about the $z$ axis. It may happen that the field obeys both reflection symmetry under time reversal and rotation symmetry under time translation.

This symmetry is not present in the HATI spectra because the exponential term which contains $S_{\mathbf{p}, \text { st }}\left(\mathbf{p}, \mathbf{k}_{\mathrm{st}} ; t, t^{\prime}\right)$ is not invariant under the reflection with respect to the $x z$ plane. In more detail, for the relative phase $\varphi=90^{\circ}$ the reflection with respect to the $x z$ plane leads to the following transformation of the OTC field and vector potential

$$
P_{x}(0) \mathbf{E}(t)=-\mathbf{E}(-t), \quad P_{x}(0) \mathbf{A}(t)=\mathbf{A}(-t),
$$

while the transformation of the stationary momentum is the same as the transformation of the OTC field. The vector potential and stationary momentum transform according to different rules under the reflection with respect to the $x z$ plane so that the term $\left[\mathbf{k}_{\mathrm{st}}+\mathbf{A}(t)\right]^{2}$ which is present in $S_{\mathbf{p}, \mathrm{st}}\left(\mathbf{p}, \mathbf{k}_{\mathrm{st}} ; t, t^{\prime}\right)$ is not invariant under this transformation and the HATI spectra do not obey this symmetry.
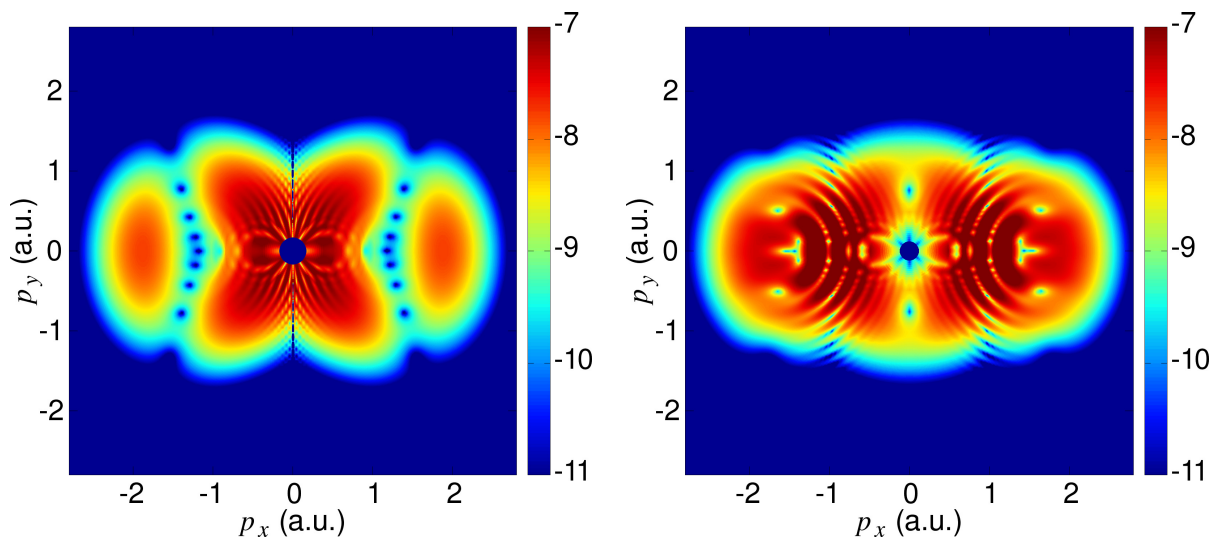

Figure 10. Logarithm of the differential ionization rate of the He atom exposed to the $\omega-2 \omega$ (left panel) and $\omega-3 \omega$ (right panel) OTC fields with the relative phase $\varphi=90^{\circ}$. Only the contribution of the direct electrons is taken into account. The spectra are to be compared with those of Figure 7 , which include both direct and rescatterred electrons. The other field parameters are the same as in Figure 6.

Before concluding this subsection, let us further elaborate on the symmetry properties of the HATI spectra generated by a few-cycle pulse. This problem is thoroughly analyzed in $[6,105]$ using the example of a linearly polarized few-cycle pulse. The HATI photoelec- 
tron momentum distribution for a long linearly polarized pulse is invariant under the reflections with respect to the $x z$ and $y z$ planes, i.e., it observes the so-called backwardforward symmetry. On the other hand, a finite pulse will never yield an ATI spectrum that exhibits strict backward-forward symmetry. Also, for very short pulses the carrier-envelope phase becomes a crucial parameter. Examples of the measured photoelectron spectra as a function of the carrier-envelope phase can be found in [123-125]. The experimental results obtained in [125] were simulated numerically using SFA and TDSE calculations. Both calculations show that the symmetry properties that are present in the angle- or energy-resolved spectra for long pulses are broken for a few-cycle pulse. The asymmetry in the photoelectron spectra is small for the low-energy electrons because these electrons quickly leave the focal plane of the laser field and travel to the detector. The time spent in the laser field is short so that the impact of the carrier-envelope phase is minor. However, the rescattered electrons spend much more time in the laser field so that the influence of the carrier-envelope phase is significant. An example of the photoelectron spectra obtained using a few-cycle pulse is given in Figure 1 of $[126,127]$. Here, the electric field is defined along the $z$ axis and we consider the spectra in the $x z$ plane. Symmetry with respect to the transformation $y \rightarrow-y$ is taken for granted. For a long linearly polarized pulse the obtained spectra would obey the reflection symmetry with respect to the transformations $z \rightarrow-z$ and $x \rightarrow-x$. However, for a few-cycle pulse the symmetry with respect to the transformation $z \rightarrow-z$ is broken, while the reflection symmetry $x \rightarrow-x$ is still present because this reflection does not change the linearly polarized field at all. The situation would be similar for an elliptically polarized field, but radically different for two-color fields. Few-cycle bicircular laser fields were considered in [128].

In conclusion, the dynamical symmetries of the driving field that involve time translation are directly mapped onto the direct and rescattered photoelectron momentum distributions. The dynamical symmetries that involve time reversal are only observed by the direct electrons. They are broken when rescattering or Coulomb effects are taken into account [129].

\subsection{Influence of the Molecular Symmetry on the Photoelectron Momentum Distribution}

Finally, let us now explore how the molecular symmetry alters the conclusions drawn above. This question has already been analyzed in detail in $[77,78]$ using the examples of homonuclear diatomic and linear polyatomic molecules exposed to a bicircular field. The same problem was investigated in [81,82] for homonuclear and heteronuclear diatomic molecules in an OTC field. The main conclusions can be summarized as follows. The symmetries of the direct and rescattered photoelectron momentum distribution that are present in the spectra for atomic targets are broken unless the molecular orbital satisfies the same symmetry properties as the driving field. To explain this in more detail we will use the examples of the bicircular and OTC fields. For the $\omega-2 \omega$ bicircular field the HATI spectrum for an atomic target is invariant with respect to a rotation by the angle $\alpha_{p}=-2 p \pi / 3$. However, the spectrum for a homonuclear diatomic molecule exposed to the same field is not invariant with respect to this rotation because the molecular orbital is only invariant with respect to a rotation by the angle $\pi$. This case is shown in Figure 3 of [77]. It is important to mention that this conclusion holds for arbitrary orientation of the molecule in the laser field. For the $\omega-3 \omega$ OTC field the atomic HATI spectrum possesses rotational symmetry by the angle $\pi$, which is also a symmetry transformation of a homonuclear diatomic molecule. As a result the same symmetry is also present in the HATI spectra obtained using a homonuclear diatomic molecule. This case is illustrated in Figure 3 of [81]. However, this symmetry is broken for a heteronuclear diatomic molecule (see Figure 2 in [82]). Finally, it is important to mention that for some molecular orientations and some values of the field parameters the spectrum can exhibit some additional rotational and/or reflection symmetries. 


\section{Conclusions}

We have performed a detailed analysis of the connection between the dynamical symmetry of the applied laser field and the selection rules for high-order harmonic generation and the symmetry properties of the photoelectron momentum distribution in (high-order) above-threshold ionization. Using the strong-field approximation we calculated the HHG and HATI spectra of atomic and molecular targets exposed to various laser fields.

We derived the selection rules for HHG of atomic targets using two different methods. One is based on the direct application of the transformation imposed by the dynamical symmetry of a particular field to the $T$-matrix element. This procedure was illustrated using elliptically polarized and OTC fields as examples. It was confirmed that for an elliptically polarized field only odd harmonics are present in the spectra. For an $r \omega-s \omega$ OTC field we have shown that, for the case where $r+s$ is even, only odd harmonics are emitted, while for $r+s$ odd both odd and even harmonics appear in the spectra.

The second method of derivation of the selection rules is based on the conservation of the projection of the total angular momentum on the quantization axis. In this case we used a bicircular field as an example. Analyzing the change of the projection of the total angular momentum on the quantization axis during the HHG process we derived the corresponding selection rule. This selection rule depends on the type of the ground state of an atom. For the $s$ ground state the magnetic quantum number is zero and the selection rule is given by Equation (30). However, for atoms with a $p$ ground state, the selection rule is different depending on whether or not the atom has a closed shell. This difference stems from the fact that for atoms with a closed shell transitions that change the magnetic quantum number are forbidden by the Pauli exclusion principle, so that Equation (30) remains valid. This is not the case if the shell is not closed and the more general rule (29) should be applied. The process in which the initial and final electron states are substates differing only in the magnetic quantum number was called degenerate combinational harmonic generation [130? ]. Both derivations were carried out for the exact field-induced dipole; we did not make use of the strong-field approximation.

We explained that, for the selection rules to be valid, the initial ground state has to be invariant under the transformation implied by the dynamical symmetry of the applied field. For atoms with an $s$ ground state this does not place any restriction on the choice of the quantization axis, while for a $p$ ground state the $z$ axis has to be chosen as the quantization axis because the $p$ state is invariant with respect to rotations around the $z$ axis. Of course, for atoms there is no preferred axis and one can always choose the quantization axis so that the mentioned selection rules are valid also for a $p$ ground state. The above method can also be applied to obtain the selection rules for other fields. The atomic selection rules are illustrated using the examples of He and Ar atoms.

After analyzing the selection rules for atomic targets, we investigated the extent to which they are violated for molecules. For molecular targets there is always a preferred axis. This is the molecular axis for diatomic and linear polyatomic molecules, while for other molecules it is the so-called principal molecular axis. For the selection rules to be applicable, the molecular ground state has to be invariant under the transformation imposed by the dynamical symmetry of the field with respect to the preferred axis. More precisely, the projection of the molecule on the laser-field polarization plane has to be invariant under this transformation. This was illustrated using numerical calculations of the HHG spectra of the $\mathrm{CO}_{2}$ and $\mathrm{O}_{3}$ molecules.

Finally, we briefly discussed the HHG process by a few-cycle pulse. In this case the harmonic spectrum depends on the carrier-envelope phase. This dependence is particularly pronounced for the plateau and the cutoff harmonics. Also, we mentioned that the position of the cutoff depends on the carrier-envelope phase. This position can be estimated using a classical model.

In the second part of this paper, we explored the symmetry properties of the ATI and HATI spectra using the examples of He and Ne atoms. The symmetries of the photoelectron momentum distribution can be traced to the behavior of the $T$-matrix element under the 
temporal and spatial transformations that are imposed by the dynamical symmetries of the driving field. The total spectra, which comprise both the direct and the rescattered electrons, observe the dynamical symmetries that are related to temporal translations. These affect the electric field and the vector potential in the same way. For example, for the OTC field with $r+s$ even the spectra possess rotational symmetry by the angle $\pi$ about the $z$ axis (perpendicular to the polarization plane of the driving field), while for $r+s$ odd the spectra possess reflection symmetry with respect to the $y z(x z)$ plane for $r$ (s) odd. Analogous results can be obtained for bicircular or other fields. In other words, for atomic targets, the dynamical symmetry of the driving field that is related to a time translation is directly mapped on the photoelectron momentum distribution. This is valid regardless of the type of the atomic ground state.

In contrast, for dynamical symmetries that imply time reversal the electric field and the vector potential transform differently. The spectrum of the direct electrons is related to the vector potential at a certain time. Hence, the spectrum of the direct electrons may observe an additional reflection symmetry, which is broken by the rescattered electrons. This is the case for bicircular fields where we discussed additional reflection symmetries with respect to several axes at certain angles with respect to the $x$ axis (see Section 4.2). For the OTC field, such additional symmetries are only present for specific relative phases between the two driving-field components. For example, for the $\omega-2 \omega$ OTC field with the relative phase $\varphi=90^{\circ}$ the direct spectrum exhibits reflection symmetries with respect to the $y z$ and $x z$ plane. The reflection symmetry with respect to the $x z$ plane is broken by the rescattering contribution. This symmetry is also broken when the Coulomb field is allowed to affect the propagation of the liberated electron. For a few-cycle pulse all of the above-mentioned symmetries are broken and the shape of the spectra strongly depends on the carrier-envelope phase.

Our approach is based, for (H)ATI, on the first two terms of the Born series, which describe the direct and the rescattered electrons. Actually, it is rather the other way around: the contributions of the first and the second term define the direct and the rescattered electrons. This is an example of a "step-by-step" approach; cf. [10]. In contrast, solutions of the TDSE and, of course, experimental data afford "all-at-once" results, which do not provide a self-evident clean-cut way to distinguish direct from rescattered electrons. Yet, this distinction is helpful in the physical interpretation. At this point, the different symmetry properties enter, which we related to different dynamical symmetries, involving either time translation or time reversal. The different symmetries of different parts of the angle-resolved spectrum allow us to identify the significance of rescattering. They also allow one to determine the effects of the Coulomb potential, which is neglected in the SFA. In general, the Coulomb effects increase the ionization rate and violate the reflection symmetry of the direct low-energy photoelectron spectra. However, they do not affect the symmetry of the medium- and high-energy photoelectron spectra nor those of the high harmonics.

Finally, we analyzed the influence of the symmetry of the molecular ground state on the photoelectron momentum distribution following the detailed analysis of our earlier papers. Briefly, the symmetry properties of the photoelectron momentum distribution obtained for atomic targets remain present in the spectra of the molecular targets provided the molecular orbital satisfies the same symmetry properties as the driving field. For example, for the $\omega-3 \omega$ OTC field the dynamical symmetry corresponds to a rotation by the angle $\pi$ about the axis perpendicular to the polarization plane. If this transformation is also a symmetry transformation of a particular molecule, then the HATI spectra obey the same symmetry properties as for atomic targets. Similar conclusions are valid for other fields.

In conclusion, we have presented a self-contained theory to obtain the selection rules for HHG and to analyze the symmetry properties of the HATI spectra in terms of the dynamical symmetries of the driving field. This theory is an alternative to other approaches, for example methods based on group theory. Numerical results were obtained applying 
the strong-field approximation including for high-order ATI the rescattering correction. In an appendix, we analyze the symmetries of the exact ionization amplitudes.

Author Contributions: All authors contributed equally to the paper. All authors have read and agreed to the published version of the manuscript.

Funding: We acknowledge support by the Ministry for Science, Higher Education and Youth, Canton Sarajevo, Bosnia and Herzegovina, and the Alexander von Humboldt Foundation.

Conflicts of Interest: The authors declare no conflict of interest.

\section{Appendix A. Symmetries of the Exact Ionization Amplitudes}

In this appendix, we will consider the symmetries of the exact ATI and HATI amplitudes, not restricted to the two lowest-order terms of the Born expansion. We will see that for dynamical symmetries that incorporate time translation the exact matrix elements obey the same symmetries as the two lowest-order (direct and rescattering) terms. However, the dynamical symmetry with time reversal generates a spatial symmetry of the angleresolved spectrum only for the lowest-order (direct) term. Higher-orders starting with the rescattering term violate this symmetry. Owing to the dominance of the direct term, we can state that a dynamical symmetry that involves time reversal generates only an approximate symmetry of the ionization amplitude.

The ionization amplitude from the field-free ground state $|0\rangle$ to a field-free continuum state $|\mathbf{p}\rangle$ with asymptotic momentum $\mathbf{p}$ reads [129]

$$
M(\mathbf{p})=\lim _{t \rightarrow \infty} \lim _{t^{\prime} \rightarrow-\infty}\left\langle\mathbf{p}\left|\mathcal{U}\left(t, t^{\prime}\right)\right| 0\right\rangle .
$$

The time-evolution operator for the electron subject to the binding potential $V(\mathbf{r})$ and the external laser field $\mathbf{E}(t)$ in the long-wavelength approximation is, in the interaction picture,

$$
\mathcal{U}\left(t, t^{\prime}\right)=\mathcal{T} \exp \left\{-i \int_{t^{\prime}}^{t} d \tau \mathcal{H}_{I}(\tau)\right\}
$$

where $\mathcal{T}$ denotes the time-ordering operator,

$$
\mathcal{H}_{I}(t)=e^{i H_{0} t} \mathbf{r} \cdot \mathbf{E}(t) e^{-i H_{0} t}
$$

is the electron-field interaction operator in length gauge and $H_{0}=\hat{\mathbf{p}}^{2} / 2+V(\mathbf{r})$ is the atomic Hamiltonian. Both $|0\rangle$ and $|\mathbf{p}\rangle$ are eigenstates of $H_{0}$.

Now, let us suppose that for some particular times $\alpha$ the field $\mathbf{E}(t)$ obeys the dynamical symmetry (an example is Equation (18) for the bicircular field)

$$
R_{\alpha} \mathbf{E}(t)=\mathbf{E}(t-\alpha), \quad R_{\alpha} \equiv R_{z}(2 \pi \alpha / T),
$$

with $T$ the period of the field. In Hilbert space, the rotation $R_{\alpha}$ is represented by the unitary operator $O_{R_{\alpha}} \equiv O_{\alpha}$ so that

$$
\left|R_{\alpha} \mathbf{p}\right\rangle=O_{\alpha}|\mathbf{p}\rangle .
$$

Then, assuming complete rotational symmetry of $H_{0}$,

$$
\begin{aligned}
O_{\alpha}^{\dagger} \mathcal{H}_{I}(\tau) O_{\alpha} & =e^{i H_{0} \tau} O_{\alpha}^{\dagger} \mathbf{r} O_{\alpha} \cdot \mathbf{E}(\tau) e^{-i H_{0} \tau}=e^{i H_{0} \tau} R_{\alpha} \mathbf{r} \cdot \mathbf{E}(\tau) e^{-i H_{0} \tau} \\
& =e^{i H_{0} \tau} \mathbf{r} \cdot R_{-\alpha} \mathbf{E}(\tau) e^{-i H_{0} \tau}=e^{i H_{0} \tau} \mathbf{r} \cdot \mathbf{E}(\tau+\alpha) e^{-i H_{0} \tau} \\
& =e^{-i H_{0} \alpha} \mathcal{H}_{I}(\tau+\alpha) e^{i H_{0} \alpha}
\end{aligned}
$$

and

$$
\int_{t^{\prime}}^{t} d \tau O_{\alpha}^{\dagger} \mathcal{H}_{I}(\tau) O_{\alpha}=e^{-i H_{0} \alpha}\left\{\int_{t^{\prime}-\alpha}^{t-\alpha} d \tau \mathcal{H}_{I}(\tau)\right\} e^{i H_{0} \alpha}
$$


Hence, finally, in the limit where $t \rightarrow \infty$ and $t^{\prime} \rightarrow-\infty$,

$$
M\left(R_{\alpha} \mathbf{p}\right)=\exp \left[-i\left(\mathbf{p}^{2} / 2+I_{p}\right) \alpha\right] M(\mathbf{p})
$$

so that the exact ionization amplitude into a continuum state with momentum $\mathbf{p}$ is invariant up to a phase upon rotation by any angle that satisfies the dynamical symmetry (A4) of the field. We showed above that if the amplitude is calculated via the strong-field approximation then this symmetry is preserved.

Now, let us consider the situation where the field $\mathbf{E}(t)$ obeys a reflection symmetry, for example, the bicircular field (12) with $r=1$ and $s=2$, which satisfies

$$
P_{x}(\pi / 3) \mathbf{E}(t)=-\mathbf{E}(T / 3-t),
$$

where $P_{x}(\beta)$ (cf. Equation (19)), generates the reflection defined below Equation (19). Now, the ionization amplitude does not observe the corresponding symmetry, i.e., $M\left(P_{x} \mathbf{p}\right)$ is not identical with $M(\mathbf{p})$ up to a phase. Namely, if one tries to redo the same proof as above, it turns out that implementation of the time-reversal symmetry (A9) turns the time-ordering of Equation (A2) into anti-time-ordering. In consequence, the ionization amplitude exhibits reflection symmetries only to first order in the Born series with respect to $V(\mathbf{r})$, where the time ordering plays no role. To second order already, time ordering is essential, since for HATI rescattering follows ionization and not vice versa.

\section{References}

1. Weyl, H. Symmetry, 1st ed.; Princeton University Press: Princeton, NJ, USA, 1952.

2. Noether, E. Invariant variation problems. Gött. Nachr. 1918, 1918, 235-257. [CrossRef]

3. Becker, W.; Grasbon, F.; Kopold, R.; Milošević, D.B.; Paulus, G.G.; Walther, H. Above-threshold ionization: From classical features to quantum effects. Adv. At. Mol. Opt. Phys. 2002, 48, 35-98. [CrossRef]

4. Milošević, D.B.; Ehlotzky, F. Scattering and reaction processes in powerful laser fields. Adv. At. Mol. Opt. Phys. 2003, 49, 373-532. [CrossRef]

5. $\quad$ Becker, A.; Faisal, F.H.M. Intense-field many-body S-matrix theory. J. Phys. B 2005, 38, R1-R56. [CrossRef]

6. Milošević, D.B.; Paulus, G.G.; Bauer, D.; Becker, W. Above-threshold ionization by few-cycle pulses. J. Phys. B 2006, 39, R203-R262. [CrossRef]

7. Lein, M. Molecular imaging using recolliding electrons. J. Phys. B 2007, 40, R135-R173. [CrossRef]

8. Krausz, F.; Ivanov, M. Attosecond physics. Rev. Mod. Phys. 2009, 81, 163-234. [CrossRef]

9. Figueira de Morisson Faria, C.; Liu, X. Electron-electron correlation in strong laser fields. J. Mod. Opt. 2011, 58, 1076-1131. [CrossRef]

10. Becker, W.; Liu, X.; Ho, P.J.; Eberly, J.H. Theories of photoelectron correlation in laser-driven multiple atomic ionization. Rev. Mod. Phys. 2012, 84, 1011-1043. [CrossRef]

11. Popruzhenko, S.V. Keldysh theory of strong field ionization: History, applications, difficulties and perspectives. J. Phys. B 2014, 47, 204001. [CrossRef]

12. Pazourek, R.; Nagele, S.; Burgdörfer, J. Attosecond chronoscopy of photoemission. Rev. Mod. Phys. 2015, 87, 765-802. [CrossRef]

13. Wolter, B.; Pullen, M.G.; Baudisch, M.; Sclafani, M.; Hemmer, M.; Senftleben, A.; Schröter, C.D.; Ullrich, J.; Moshammer, R.; Biegert, J. Strong-field physics with mid-IR fields. Phys. Rev. X 2015, 5, 021034. [CrossRef]

14. Becker, W.; Goreslavski, S.P.; Milošević, D.B.; Paulus, G.G. The plateau in above-threshold ionization: The keystone of rescattering physics. J. Phys. B 2018, 51, 162002. [CrossRef]

15. Ben-Tal, N.; Moiseyev, N.; Beswick, A. The effect of Hamiltonian symmetry on generation of odd and even harmonics. J. Phys. B 1993, 26, 3017-3024. [CrossRef]

16. Long, S.; Becker, W.; McIver, J.K. Model calculations of polarization-dependent two-color high-harmonic generation. Phys. Rev. A 1995, 52, 2262-2278. [CrossRef] [PubMed]

17. Alon, O.E.; Averbukh, V.; Moiseyev, N. Selection rules for the high harmonic generation spectra. Phys. Rev. Lett. 1998, 80, 3743-3746. [CrossRef]

18. Ceccherini, F.; Bauer, D.; Cornolti, F. Dynamical symmetries and harmonic generation. J. Phys. B 2001, 34, 5017-5029. [CrossRef]

19. Zuo, T.; Bandrauk, A.D. High-order harmonic generation in intense laser and magnetic fields. J. Nonlinear Opt. Phys. Mat. 1995, 4, 533-546. [CrossRef]

20. Milošević, D.B.; Becker, W.; Kopold, R. Generation of circularly polarized high-order harmonics by two-color coplanar field mixing. Phys. Rev. A 2000, 61, 063403. [CrossRef]

21. Yue, S.; Brennecke, S.; Du, H.; Lein, M. Probing dynamical symmetries by bicircular high-order harmonic spectroscopy beyond the Born-Oppenheimer approximation. Phys. Rev. A 2020, 101, 053438. [CrossRef] 
22. Tong, X.-M.; Chu, S.-I. Generation of circularly polarized multiple high-order harmonic emission from two-color crossed laser beams. Phys. Rev. A 1998, 58, R2656-R2659. [CrossRef]

23. Fleischer, A.; Moiseyev, N. Attosecond laser pulse synthesis using bichromatic high-order harmonic generation. Phys. Rev. A 2006, 74, 053806. [CrossRef]

24. Milošević, D.B. Circularly polarized high harmonics generated by a bicircular field from inert atomic gases in the $p$ state: A tool for exploring chirality-sensitive processes. Phys. Rev. A 2015, 92, 043827. [CrossRef]

25. Milošević, D.B. High-order harmonic generation by a bichromatic elliptically polarized field: Conservation of angular momentum. J. Phys. B 2015, 48, 171001. [CrossRef]

26. Odžak, S.; Hasović, E.; Milošević, D.B. Strong-field-approximation theory of high-order harmonic generation by polyatomic molecules. Phys. Rev. A 2016, 93, 043413. [CrossRef]

27. Hasović, E.; Odžak, S.; Becker, W.; Milošević, D.B. High-order harmonic generation in non-planar molecules driven by a bicircular field. Mol. Phys. 2017, 115, 1750-1757. [CrossRef]

28. Liu, X.; Zhu, X.; Li, L.; Li, Y.; Zhang, Q.; Lan, P.; Lu, P. Selection rules of high-order-harmonic generation: Symmetries of molecules and laser fields. Phys. Rev. A 2016, 94, 033410. [CrossRef]

29. Habibović, D.; Becker, W.; Milošević, D.B. High-order harmonic generation by planar polyatomic molecules exposed to an orthogonally polarized two-color laser field. J. Phys. B 2021, 54, 134004. [CrossRef]

30. Neufeld, O.; Podolsky, D.; Cohen, O. Floquet group theory and its application to selection rules in harmonic generation. Nat. Commun. 2019, 10, 405. [CrossRef]

31. Ghimire, S.; DiChiara, A.D.; Sistrunk, E.; Agostini, P.; DiMauro, L.F.; Reis, D.A. Observation of high-order harmonic generation in a bulk crystal. Nat. Phys. 2011, 7, 138-141. [CrossRef]

32. Moiseyev, N. Selection rules for harmonic generation in solids. Phys. Rev. A 2015, 91, 053811. [CrossRef]

33. Reiss, H.R. Effect of an intense electromagnetic field on a weakly bound system. Phys. Rev. A 1980, 22, 1786-1813. [CrossRef]

34. Mittleman, M.H. Introduction to the Theory of Laser-Atom Interactions, 2nd ed.; Plenum Press: New York, NY, USA, 1993.

35. Delone, N.B.; Krainov, V.P. Multiphoton Processes in Atoms, 2nd ed.; Springer: Berlin, Germany, 2000.

36. DiMauro, L.F.; Agostini, P. Ionization dynamics in strong laser fields. Adv. At. Mol. Opt. Phys. 1995, 35, 79-120. [CrossRef]

37. Muller, H.G.; Agostini, P.; Petite, G. Multiphoton ionization. In Atoms in Intense Laser Fields, 1st ed.; Gavrila, M., Ed.; Academic: New York, NY, USA, 1992; pp. 1-43.

38. Milošević, D.B. Strong-field approximation for ionization of a diatomic molecule by a strong laser field. Phys. Rev. A 2006, 74, 063404. [CrossRef]

39. Schafer, K.J.; Yang, B.; DiMauro, L.F.; Kulander, K.C. Above threshold ionization beyond the high harmonic cutoff. Phys. Rev. Lett. 1993, 70, 1599-1602. [CrossRef]

40. Yang, B.; Schafer, K.J.; Walker, B.; Kulander, K.C.; Agostini, P.; DiMauro, L.F. Intensity-dependent scattering rings in high order above-threshold ionization. Phys. Rev. Lett. 1993, 71, 3770-3773. [CrossRef]

41. Paulus, G.G.; Nicklich, W.; Xu, H.; Lambropoulos, P.; Walther, H. Plateau in above threshold ionization spectra. Phys. Rev. Lett. 1994, 72, 2851-2854. [CrossRef]

42. Paulus, G.G.; Nicklich, W.; Walther, H. Investigation of above-threshold ionization with femtosecond pulses: Connection between plateau and angular distribution of the photoelectrons. Europhys. Lett. 1994, 27, 267-272. [CrossRef]

43. Paulus, G.G.; Nicklich, W.; Zacher, F.; Lambropoulos, P.; Walther, H. High-order above-threshold ionization of atomic hydrogen using intense, ultrashort laser pulses. J. Phys. B 1996, 29, L249-L256. [CrossRef]

44. Walker, B.; Sheehy, B.; Kulander, K.C.; DiMauro, L.F. Elastic rescattering in the strong field tunneling limit. Phys. Rev. Lett. 1996, 77, 5031-5034. [CrossRef] [PubMed]

45. Becker, W.; Lohr, A.; Kleber, M. Effects of rescattering on above-threshold ionization. J. Phys. B 1994, 27, L325-L332; Erratum in J. Phys. B 1995, 28, 1931. [CrossRef]

46. Lohr, A.; Kleber, M.; Kopold, R.; Becker, W. Above-threshold ionization in the tunneling regime. Phys. Rev. A 1996, 55, R4003-R4006. [CrossRef]

47. Milošević, D.B.; Ehlotzky, F. Coulomb and rescattering effects in above-threshold ionization. Phys. Rev. A 1998, 58, 3124-3127. [CrossRef]

48. Milošević, D.B.; Ehlotzky, F. S-matrix theory of above-threshold ionization in a bichromatic laser field. J. Phys. B 1999, 32, 1585-1596. [CrossRef]

49. Kopold, R.; Milošević, D.B., Becker, W. Rescattering processes for elliptical polarization: A quantum trajectory analysis. Phys. Rev. Lett. 2000, 84, 3831-3834. [CrossRef] [PubMed]

50. Salières, P.; Carré, B.; Le Déroff, L.; Grasbon, F.; Paulus, G.G.; Walther, H.; Kopold, R.; Becker, W.; Milošević, D.B.; Sanpera, A.; et al. Feynman's path-integral approach for intense-laser-atom interactions. Science 2001, 292, 902-905. [CrossRef]

51. Paulus, G.G.; Becker, W.; Walther, H. Classical rescattering effects in two-color above-threshold ionization. Phys. Rev. A 1995, 52, 4043-4053. [CrossRef]

52. Ehlotzky, F. Atomic phenomena in bichromatic laser fields. Phys. Rep. 2001, 345, 176-264. [CrossRef]

53. Hasović, E.; Milošević, D.B.; Becker, W. A method of carrier-envelope phase control for few-cycle laser pulses. Las. Phys. Lett. 2006, 3, 200-204. [CrossRef] 
54. Kramo, A.; Hasović, E.; Milošević, D.B.; Becker, W. Above-threshold detachment by a two-color bicircular laser field. Las. Phys. Lett. 2007, 4, 279-286. [CrossRef]

55. Hasović, E.; Kramo, A.; Milošević, D.B. Energy- and angle-resolved photoelectron spectra of above-threshold ionization and detachment. Eur. Phys. J. D 2007, 160, 205-216. [CrossRef]

56. Mancuso, C.A.; Hickstein, D.D.; Grychtol, P.; Knut, R.; Kfir, O.; Tong, X.-M.; Dollar, F.; Zusin, D.; Gopalakrishnan, M.; Gentry, C.; et al. Strong-field ionization with two- color circularly polarized laser fields. Phys. Rev. A 2015, 91, 031402(R). [CrossRef]

57. Hasović, E.; Becker, W.; Milošević, D.B. Electron rescattering in a bicircular laser field. Opt. Express 2016, 24, 6413-6424. [CrossRef]

58. Mancuso, C.A.; Hickstein, D.D.; Dorney, K.M.; Ellis, J.L.; Hasović, E.; Knut, R.; Grychtol, P.; Gentry, C.; Gopalakrishnan, M.; Zusin, D.; et al. Controlling electron-ion rescattering in two-color circularly polarized femtosecond laser fields. Phys. Rev. A 2016, 93, 053406. [CrossRef]

59. Milošević, D.B.; Becker, W. Improved strong-field approximation and quantum-orbit theory: Application to ionization by a bicircular laser field. Phys. Rev. A 2016, 93, 063418. [CrossRef]

60. Posthumus, J.H. The dynamics of small molecules in intense laser fields. Rep. Prog. Phys. 2004, 67, 623-665. [CrossRef]

61. Stapelfeldt, H.; Seideman, M. Colloquium: Aligning molecules with strong laser pulses. Rev. Mod. Phys. 2005, 75, 543-557. [CrossRef]

62. Talebpour, A.; Chien, C.-Y.; Chin, S.L. The effects of dissociative recombination in multiphoton ionization of $\mathrm{O}_{2}$. J. Phys. B 1996, 29, L677-L680. [CrossRef]

63. Talebpour, A.; Larochelle, S.; Chin, S.L. Suppressed tunnelling ionization of the $\mathrm{D}_{2}$ molecule in an intense Ti:sapphire laser pulse. J. Phys. B 1998, 31, L49-L58. [CrossRef]

64. Guo, C.; Li, M.; Nibarger, J.P.; Gibson, G.N. Single and double ionization of diatomic molecules in strong laser fields. Phys. Rev. A 1998, 58, R4271-R4274. [CrossRef]

65. DeWitt, M.J.; Wells, E.; Jones, R.R. Ratiometric comparison of intense field ionization of atoms and diatomic molecules. Phys. Rev. Lett. 2001, 87, 153001. [CrossRef]

66. Wells, E.; DeWitt, M.J.; Jones, R.R. Comparison of intense-field ionization of diatomic molecules and rare-gas atoms. Phys. Rev. A 2002, 66, 013409. [CrossRef]

67. Grasbon, F.; Paulus, G.G.; Chin, S.L.; Walther, H.; Muth-Böhm, J.; Becker, A.; Faisal, F.H.M. Signatures of symmetry-induced quantum-interference effects observed in above-threshold-ionization spectra of molecules. Phys. Rev. A 2001, 63, 041402(R). [CrossRef]

68. Litvinyuk, I.V.; Lee, K.F.; Dooley, P.W.; Rayner, D.M.; Villeneuve, D.M.; Corkum, P.B. Alignment-dependent strong field ionization of molecules. Phys. Rev. Lett. 2003, 90, 233003. [CrossRef]

69. Eremina, E.; Liu, X.; Rottke, H.; Sandner, W.; Schätzel, M.G.; Dreischuh, A.; Paulus, G.G.; Walther, H.; Moshammer, R.; Ullrich, J. Influence of molecular structure on double ionization of $\mathrm{N}_{2}$ and $\mathrm{O}_{2}$ by high intensity ultrashort laser pulses. Phys. Rev. Lett. 2004, 92, 173001. [CrossRef]

70. Ren, X.; Zhang, J.; Liu, P.; Wang, Y.; Xu, Z. Photoelectron angular distributions of molecules in bichromatic laser fields of circular polarization. Phys. Lett. A 2009, 373, 227-230. [CrossRef]

71. Fernández, J.; Madsen, B. Above-threshold ionization, energy-resolved photoelectron angular distributions, and momentum distributions of $\mathrm{H}_{2}{ }^{+}$in intense femtosecond laser pulses. Phys. Rev. A 2009, 79, 063406. [CrossRef]

72. Yuan, K.-J.; Bandrauk, A. Molecular above-threshold-ionization angular distributions with intense circularly polarized attosecond XUV laser pulses. Phys. Rev. A 2012, 85, 053419. [CrossRef]

73. Fetić, B.; Milošević, D.B. High-order above-threshold ionization of the $\mathrm{H}_{2}{ }^{+}$ion: The role of internuclear distance. Phys. Rev. A 2019, 99, 043426. [CrossRef]

74. Busuladžić, M.; Gazibegović-Busuladžić, A.; Milošević, D.B.; Becker, W. Angle-resolved high-order above-threshold ionization of a molecule: Sensitive tool for molecular characterization. Phys. Rev. Lett. 2008, 100, 203003. [CrossRef]

75. Busuladžić, M.; Gazibegović-Busuladžić, A.; Milošević, D.B.; Becker, W. Strong-field approximation for ionization of a diatomic molecule by a strong laser field. II. The role of electron rescattering off the molecular centers. Phys. Rev. A 2008, 78, 033412. [CrossRef]

76. Busuladžić, M.; Gazibegović-Busuladžić, A.; Milošević, D.B. Strong-field approximation for ionization of a diatomic molecule by a strong laser field. III. High-order above-threshold ionization by an elliptically polarized field. Phys. Rev. A 2009, 80, 013420. [CrossRef]

77. Busuladžić, M.; Gazibegović-Busuladžić, A.; Milošević, D.B. Strong-field ionization of homonuclear diatomic molecules by a bicircular laser field: Rotational and reflection symmetries. Phys. Rev. A 2017, 95, 033411. [CrossRef]

78. Gazibegović-Busuladžić, A.; Busuladžić, M.; Hasović, E.; Becker, W.; Milošević, D.B. Strong-field ionization of linear molecules by a bicircular laser field: Symmetry considerations. Phys. Rev. A 2018, 97, 043432. [CrossRef]

79. Gazibegović-Busuladžić, A.; Busuladžić, M.; Čerkić, A.; Hasović, E.; Becker, W.; Milošević, D.B. Strong-field ionization of linear molecules by a bichromatic elliptically polarized laser field with coplanar counterrotating or corotating components of different frequencies. J. Phys. Conf. Ser. 2019, 1206, 012003. [CrossRef]

80. Busuladžić, M.; Čerkić, A.; Gazibegović-Busuladžić, A.; Hasović, E.; Milošević, D.B. Molecular-orientation-dependent interference and plateau structures in strong-field ionization of a diatomic molecule by a corotating bichromatic elliptically polarized laser field. Phys. Rev. A 2018, 98, 013413. [CrossRef] 
81. Habibović, D.; Gazibegović-Busuladžić, A.; Busuladžić, M.; Čerkić, A.; Milošević, D.B. Strong-field ionization of homonuclear diatomic molecules using orthogonally polarized two-color laser fields. Phys. Rev. A 2020, 102, 023111. [CrossRef]

82. Habibović, D.; Gazibegović-Busuladžić, A.; Busuladžić, M.; Milošević, D.B. Strong-field ionization of heteronuclear diatomic molecules using an orthogonally polarized two-color laser field. Phys. Rev. A 2021, 103, 053101. [CrossRef]

83. L'Huillier, A.; Schafer, K.J.; Kulander, K. Theoretical aspects of intense field harmonic generation. J. Phys. B 1991, $24,3315-3341$. [CrossRef]

84. Bauer, D. (Ed.) Computational Strong-Field Quantum Dynamics: Intense Light-Matter Interactions; De Gruyter Textbook: Berlin, Germany, 2016.

85. Fetić, B.; Milošević, D.B. Numerical solution of the time-dependent Schrödinger equation for $\mathrm{H}_{2}^{+}$ion with application to high-harmonic generation and above-threshold ionization. Phys. Rev. E 2017, 95, 053309. [CrossRef]

86. Clarke, D.D.A.; Armstrong, G.S.J.; Brown, A.C.; van der Hart, H.W. R-matrix-with-time-dependence theory for ultrafast atomic processes in arbitrary light fields. Phys. Rev. A 2018, 98, 053442. [CrossRef]

87. Odžak, S.; Hasović, E.; Milošević, D.B. High-order harmonic generation in polyatomic molecules induced by a bicircular laser field. Phys. Rev. A 2016, 94, 033419. [CrossRef]

88. Odžak, S.; Milošević, D.B. Interference effects in high-order harmonic generation by homonuclear diatomic molecules. Phys. Rev. A 2009, 79, 023414. [CrossRef]

89. Levine, I.N. Quantum Chemistry, 5th ed.; Prentice-Hall: Upper Saddle River, NJ, USA, 2000.

90. Schmidt, M.W.; Baldridge, K.K.; Boatz, J.A.; Elbert, S.T.; Gordon, M.S.; Jensen, J.H.; Koseki, S.; Matsunaga, N.; Nguyen, K.A.; Su, S.; et al. General atomic and molecular electronic structure system. J. Comput. Chem. 1993, 14, 1347-1363. [CrossRef]

91. Hasović, E.; Milošević, D.B. Strong-field approximation for above-threshold ionization of polyatomic molecules. Phys. Rev. A 2012, 86, 043429. [CrossRef]

92. Hasović, E.; Milošević, D.B. Strong-field approximation for above-threshold ionization of polyatomic molecules. II. The role of electron rescattering off the molecular centers. Phys. Rev. A 2014, 89, 053401. [CrossRef]

93. Gallagher, T.F. Above-threshold ionization in the low-frequency limit. Phys. Rev. Lett. 1988, 61, 2304-2307. [CrossRef] [PubMed]

94. Corkum, P.B.; Burnett, N.H.; Brunel, F. Above-threshold ionization in the long-wavelength limit. Phys. Rev. Lett. 1989, 62, 1259-1262. [CrossRef]

95. Corkum, P.B. Plasma perspective on strong field multiphoton ionization. Phys. Rev. Lett. 1993, 71, 1994-1997. [CrossRef]

96. Milošević, D.B.; Becker, W. X-ray harmonic generation by orthogonally polarized two-color fields: Spectral shape and polarization. Phys. Rev. A 2019, 100, 031401(R). [CrossRef]

97. Habibović, D.; Milošević, D.B. Ellipticity of high-order harmonics generated by aligned homonuclear diatomic molecules exposed to an orthogonal two-color laser field. Photonics 2020, 7, 110. [CrossRef]

98. Smirnova, O.; Spanner, M.; Ivanov, M. Anatomy of strong field ionization II: to dress or not to dress? J. Mod. Opt. 2007, 54, 1019-1038. [CrossRef]

99. Becker, W.; Chen, J.; Chen, S.G.; Milošević, D.B. Dressed-state strong-field approximation for laser-induced molecular ionization. Phys. Rev. A 2007, 76, 033403. [CrossRef]

100. Okunishi, M.; Itaya, R.; Shimada, K.; Prümper, G.; Ueda, K.; Busuladžić, M.; Gazibegović-Busuladžić, A.; Milošević, D.B.; Becker, W. Angle-resolved high-order above-threshold ionization spectra for $\mathrm{N}_{2}$ and $\mathrm{O}_{2}$ : Measurements and the strong-field approximation. J. Phys. B 2008, 41, 201004. [CrossRef]

101. Okunishi, M.; Itaya, R.; Shimada, K.; Prümper, G.; Ueda, K.; Busuladžić, M.; Gazibegović-Busuladžić, A.; Milošević, D.B.; Becker, W. Two-source double-slit interference in angle-resolved high-energy above-threshold ionization spectra of diatoms. Phys. Rev. Lett. 2009, 103, 043001. [CrossRef]

102. Busuladžić, M.; Milošević, D.B. Simulation of the above-threshold-ionization experiment using the molecular strong-field approximation: The choice of gauge. Phys. Rev. A 2010, 82, 015401. [CrossRef]

103. Gazibegović-Busuladžić, A.; Hasović, E.; Busuladžić, M.; Milošević, D.B.; Kelkensberg, F.; Siu, W.K.; Vrakking, M.J.J.; Lépine, F.; Sansone, G.; Nisoli, M.; et al. Above-threshold ionization of diatomic molecules by few-cycle laser pulses. Phys. Rev. A 2011, 84, 043426. [CrossRef]

104. Quan, W.; Lai, X.Y.; Chen, Y.J.; Wang, C.L.; Hu, Z.L.; Liu, X.J.; Hao, X.L.; Chen, J.; Hasović, E.; Busuladžić, M.; et al. Resonancelike enhancement in high-order above-threshold ionization of molecules. Phys. Rev. A 2013, 88, 021401(R). [CrossRef]

105. Milošević, D.B. Few-cycle-laser-pulse induced and assisted processes in atoms, molecules, and nanostructures. In Ultrafast Dynamics Driven by Intense Light Pulses: From Atoms to Solids, from Lasers to Intense X-rays, 1st ed.; Kitzler, M., Gräfe, S., Eds.; Springer: Berlin, Germany, 2016; Volume 86, pp. 27-48.

106. Kübel, M.; Wustelt, P.; Zhang, Y.; Skruszewicz, S.; Hoff, D.; Würzler, D.; Kang, H.; Zille, D.; Adolph, D.; Paulus, G.G.; et al. High-order phase-dependent asymmetry in the above-threshold ionization plateau. Phys. Rev. Lett. 2021, 126, 113201. [CrossRef] [PubMed]

107. Milošević, D.B.; Becker, W. High-order harmonic generation by bi-elliptical orthogonally polarized two-color fields. Phys. Rev. A 2020, 102, 023107. [CrossRef]

108. Mauger, F.; Bandrauk, A.D.; Uzer, T. Circularly polarized molecular high harmonic generation using a bicircular laser. J. Phys. B 2016, 49, 10LT01. [CrossRef] 
109. Milošević, D.B. Cut-off law for high-harmonic generation by an elliptically polarized laser field. J. Phys. B 2000, 33, $2479-2488$. [CrossRef]

110. Milošević, D.B.; Becker, W.; Kopold, R. High-harmonic generation by two-color circularly polarized field mixing. In Atoms, Molecules and Quantum Dots in Laser Fields: Fundamental Processes; Bloembergen, N., Rahman, N., Rizzo A., Eds.; Società Italiana di Fisica: Bologna, Italy, 2001; Volume 71, pp. 239-252.

111. Milošević, D.B.; Becker, W. Generation of elliptically polarized soft $\mathrm{x}$ rays using high-order harmonic generation with orthogonal two-color laser fields. J. Phys. Conf. Ser. 2020, 1508, 012001. [CrossRef]

112. Birkeland, T.; Førre, M.; Hansen, J.P.; Selstø, S. Dynamics of H(2p) ionization in ultrashort strong laser pulses. J. Phys. B 2004, 37, 4205-4219. [CrossRef]

113. Habibović, D.; Becker, W.; Milošević, D.B. Attosecond-pulse-train generation using an orthogonally polarized two-color laser field. J. Opt. Soc. Am. B 2021, submitted.

114. Milošević, D.B. Generation of elliptically polarized attosecond pulse trains. Opt. Lett. 2015, 40, 2381-2384. [CrossRef]

115. Medišauskas, L.; Wragg, J.; van der Hart, H.W.; Ivanov, M.Y. Generating isolated elliptically polarized attosecond pulses using bichromatic counterrotating circularly polarized laser fields. Phys. Rev. Lett. 2015, 115, 153001. [CrossRef]

116. De Bohan, A.; Antoine, P.; Milošević, D.B.; Piraux, B. Phase-dependent harmonic emission with ultrashort laser pulses. Phys. Rev. Lett. 1998, 81, 1837-1840. [CrossRef]

117. Baltuška, A.; Udem, T.; Uiberacker, M.; Hentschel, M.; Goulielmakis, E.; Gohle, C.; Holzwarth, R.; Yakovlev, V.S.; Scrinzi, A.; Hänsch, T.W.; et al. Attosecond control of electronic processes by intense light fields. Nature 2003, 421, 611-615. [CrossRef]

118. Möller, M.; Meyer, F.; Sayler, A.M.; Paulus, G.G.; Kling, M.F.; Schmidt, B.E.; Becker, W.; Milošević, D.B. Off-axis low-energy structures in above-threshold ionization. Phys. Rev. A 2014, 90, 023412. [CrossRef]

119. Huismans, Y.; Rouzée, A.; Gijsbertsen, A.; Jungmann, J.H.; Smolkowska, A.S.; Logman, P.S.W.M.; Lépine, F.; Cauchy, C.; Zamith, S.; Marchenko, T.; et al. Time-resolved holography with photoelectrons. Science 2011, 331, 61-64. [CrossRef]

120. Hickstein, D.D.; Ranitovic, P.; Witte, S.; Tong, X.-M.; Huismans, Y.; Arpin, P.; Zhou, X.; Keister, K.E.; Hogle, C.W.; Zhang, B.; et al. Direct visualization of laser-driven electron multiple scattering and tunneling distance in strong-field ionization. Phys. Rev. Lett. 2012, 109, 073004. [CrossRef] [PubMed]

121. Figueira de Morisson Faria, C.; Maxwell, A.S. It is all about phases: Ultrafast holographic photoelectron imaging. Rep. Prog. Phys. 2020, 83, 034401. [CrossRef] [PubMed]

122. Li, M.; Jiang, W.-C.; Xie, H.; Luo, S.; Zhou, Y.; Lu, P. Strong-field photoelectron holography of atoms by bicircular two-color laser pulses. Phys. Rev. A 2018, 97, 023415. [CrossRef]

123. Lindner, F.; Schätzel, M.G.; Walther, H.; Baltuška, A.; Goulielmakis, E.; Krausz, F.; Milošević, D.B.; Bauer, D.; Becker, W.; Paulus, G.G. Attosecond double-slit experiment. Phys. Rev. Lett. 2005, 95, 040401. [CrossRef]

124. Paulus, G.G.; Grasbon, F.; Walther, H.; Villoresi, P.; Nisoli, M.; Stagira, S.; Priori, E.; De Silvestri, S. Absolute-phase phenomena in photoionization with few-cycle laser pulses. Nature 2001, 414, 182-184. [CrossRef]

125. Kling, M.F.; Rauschenberger, J.; Verhoef, A.J.; Hasović, E.; Uphues, T.; Milošević, D.B.; Muller, H.G.; Vrakking, M.J.J. Imaging of carrier-envelope phase effects in above-threshold ionization with intense few-cycle laser fields. New J. Phys. 2008, 10, 025024. [CrossRef]

126. Milošević, D.B.; Paulus, G.G.; Becker, W. High-order above-threshold ionization with few-cycle pulse: A meter of the absolute phase. Opt. Express 2003, 11, 1418-1429. [CrossRef]

127. Milošević, D.B.; Hasović, E.; Odžak, S.; Busuladžić, M.; Gazibegović-Busuladžić, A.; Becker, W. New results in above-threshold ionization and high-order harmonic generation of atomic and molecular systems. Las. Phys. 2009, 19, 185-190. [CrossRef]

128. Hoang, V.-H.; Le, V.-H.; Lin, C.D.; Le, A.-T. Retrieval of target structure information from laser-induced photoelectrons by few-cycle bicircular laser fields. Phys. Rev. A 2017, 95, 031402. [CrossRef]

129. Becker, W.; Kleber, M.; Lohr, A.; Paulus, G.G.; Walther, H.; Zacher, F. Electron spectra of above-threshold ionization in elliptically polarized laser fields. Las. Phys. 1998, 8, 56-68.

130. Ostrovsky, V.N. High harmonic generation by anions and atoms: Effect of initial/final-state wavefunctions. J. Phys. B 2005, 38, 4399-4406. [CrossRef] 